\title{
All quiet in the outer halo: chemical abundances in the globular cluster Pal $3^{\star, \star \star}$
}

\author{
A. Koch ${ }^{1}$, P. Côté ${ }^{2}$, and A. McWilliam ${ }^{3}$ \\ ${ }^{1}$ Department of Physics \& Astronomy, University of Leicester, University Road, Leicester LE1 7RH, UK \\ e-mail: ak326@astro.le.ac.uk \\ 2 National Research Council of Canada, Herzberg Institute of Astrophysics, 5071 West Saanich Road, Victoria, BC V9E 2E7, Canada \\ 3 Carnegie Observatories, 813 Santa Barbara St., Pasadena, CA 91101, USA
}

Received 3 July 2009 / Accepted 18 August 2009

ABSTRACT

\begin{abstract}
Context. Globular clusters (GCs) in the outer halo are important probes of the composition and origin of the Galactic stellar halo. Aims. We derive chemical element abundance ratios in red giants belonging to the remote $(R \sim 90 \mathrm{kpc}) \mathrm{GC}$ Pal 3 and compare our measurements to those for red giant stars in both inner and outer halo GCs.

Methods. From high-resolution spectroscopy of four red giants, obtained with the Magellan/MIKE spectrograph at moderately high $S / N$, we derive chemical abundances for $25 \alpha$-, iron peak-, and neutron-capture elements. These abundance ratios are confirmed by co-adding low $S / N$ HIRES spectra of 19 stars along the red giant branch.

Results. Pal 3 shows $\alpha$-enhanced abundance patterns, and also its Fe-peak and neutron-capture element ratios, are fully compatible with those found in halo field stars and representative inner halo GCs of the same metallicity (such as M 13). The heavy elements in $\mathrm{Pal} 3$ appear to be governed by $r$-process nucleosynthesis. Our limited sample does not show any significant star-to-star abundance variations in this cluster, although a weak Na-O anti-correlation cannot be ruled out by the present data.

Conclusions. Pal 3 thus appears as an archetypical GC with abundance ratios dissimilar to dwarf spheroidal stars, ruling out a direct connection to such external systems. This conclusion is underscored by the lack of significant abundance spreads in this GC, in contrast to the broad abundance distributions seen in the dwarf galaxies. Pal 3 appears to have evolved chemically in analogy to the majority of GCs belonging to the Galactic inner and outer halo, experiencing a similar enrichment history.
\end{abstract}

Key words. stars: abundances - Galaxy: abundances - Galaxy: evolution - Galaxy: halo - globular clusters: individual: Pal 3

\section{Introduction}

As the oldest stellar systems in the universe, globular clusters (GCs) bear the imprints of the early formation and evolution epochs of the Milky Way (MW) system. In particular, the absence of a metallicity gradient in the outer halo ${ }^{1}$ led to the notion of an accretion origin for the Galactic stellar halo that extended over several Gyr (Searle \& Zinn 1978). The separation into inner and outer halo populations has now been firmly established for both field stars and the GCs (e.g., Hartwick 1987; Norris \& Ryan 1989; Preston et al. 1991; Kinman et al. 1994; Carney et al. 1996; Chiba \& Beers 2000; Carollo et al. 2007; Lee et al. 2007; Miceli et al. 2008). This scenario is supported by the

\footnotetext{
* This paper includes data gathered with the $6.5 \mathrm{~m}$ Magellan Telescopes located at Las Campanas Observatory, Chile. Some of the data presented herein were obtained at the W. M. Keck Observatory, which is operated as a scientific partnership among the California Institute of Technology, the University of California and the National Aeronautics and Space Administration. The Observatory was made possible by the generous financial support of the W. M. Keck Foundation.

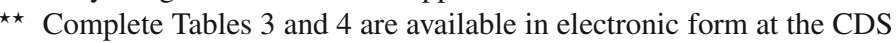
via anonymous ftp to cdsarc.u-strasbg.fr $(130.79 .128 .5)$ or via http://cdsweb.u-strasbg.fr/cgi-bin/qcat?J/506/729

1 Many suggestions for the radius at which the outer halo separates from the inner halo are given in the literature, ranging from 8 to $30 \mathrm{kpc}$. The precise choice does not matter for Pal 3 since, at $R \sim 90 \mathrm{kpc}$, it is undoubtedly a member of the outer halo.
}

existence of a pronounced second-parameter problem among the outer halo GCs (Catelan et al. 2001) which points to a broad age range within this population. Of prime importance are the chemical abundance patterns of halo field and GC stars (Freeman \& Bland-Hawthorne 2002; Pritzl et al. 2005). These are key observables that allow intercomparisons of the GCs to the dwarf spheroidal (dSph) galaxies (which are thought to have been accreted into the halo) and enable tests for (in)homogeneities among the inner and outer GC systems.

Pal 3 is a faint $\left(M_{V} \sim-5.7 \mathrm{mag}\right)$ outer halo GC and, at a Galactocentric distance of $\sim 92 \mathrm{kpc}$ (Stetson et al. 1999; Hilker 2006), one of only six known halo GCs at distances of $\sim 100 \mathrm{kpc}$ or beyond. It is one of the most spatially-extended GCs, similar to the most compact ultrafaint dSph (or most extended star cluster) candidates Willman I (Willman et al. 2005) or Segue I (Belokurov et al. 2007). At the same time, it is comparable in magnitude to the ultrafaint Leo IV (Belokurov et al. 2007) or Ursa Major I (Zucker et al. 2006) systems, and therefore falls close to the gap between the dSphs and GCs in the magnituderadius diagram (e.g., Gilmore et al. 2007). Lacking kinematic information, extended GCs like Pal 3 are therefore sometimes considered to be possible low-luminosity galaxies.

Pal 3 is not part of any currently known stream (e.g., Palma et al. 2002) and it can be firmly excluded as member of the Sagittarius system (e.g, Bellazzini et al. 2003). Interestingly, within the large uncertainty of its proper motion, its orbit is 
compatible both with being bound or unbound to the MW. Thus, it is conceivable that it has been captured by the Galaxy and is falling onto the MW for the first time (see also Chapman et al. 2007).

Although the age estimates in the literature do not always agree (e.g., Stetson et al. 1999 vs. Vandenberg 2000), it seems clear that Pal 3 represents a halo GC, similar to the SMC cluster NGC 121 (Glatt et al. 2008), and that Pal 3 is likely 1-2 Gyr younger than inner halo GCs of the same metallicity such as M 3 and M 13 (e.g., Cohen \& Meléndez 2005a). An accurate age derivation, however, hinges on the assumption that "they [Pal 3 and M3/M13] truly are chemically indistinguishable" (Vandenberg 2000). In this spirit, Cohen \& Meléndez (2005b) found that the outer halo GC NGC $7492\left(R_{\mathrm{GC}}=25 \mathrm{kpc}\right)$ has experienced a very similar enrichment history to the inner halo GCs, such as M3 or M13; in terms of their chemical abundances, these populations appear to be similar.

All previous spectroscopic studies on Pal 3 have been carried out in low-dispersion mode (Ortolani \& Gratton 1989) and using the calcium triplet (CaT) metallicity indicator (Armandroff et al. 1992). Although no high-dispersion abundance study has been carried out for this remote cluster to place it in the context of the accretion scenario, low-dispersion spectroscopy and colour magnitude diagram (CMD) studies have already established Pal 3 as a mildly metal poor system, with $[\mathrm{Fe} / \mathrm{H}]$ estimates ranging from -1.57 to -1.8 dex (Ortolani \& Gratton 1989; Armandroff et al. 1992; Stetson et al. 1999; Kraft \& Ivans 2003; Hilker 2006). In this paper, we aim to extend the chemical element information for objects in the Galactic halo out to larger distances, and to present an initial characterisation of Pal 3's chemical abundance patterns.

\section{Data}

\subsection{HIRES spectra}

During three nights in February and March 1999, we observed 25 stars in Pal 3 using the HIRES echelle spectrograph (Vogt et al. 1994) on the Keck I telescope. Our targets for this run were selected from a CMD constructed from $B V$ imaging obtained with the Low-Resolution Imaging Spectrometer (LRIS; Oke et al. 1995) on the night of 13 January 1999. A CMD reaching roughly one magnitude below the main-sequence turnoff was constructed using short and long exposures in both bandpasses $(60 \mathrm{~s}+3 \times 180 \mathrm{~s}$ in $V$, and $240 \mathrm{~s}+3 \times 420 \mathrm{~s}$ in $B)$.

HIRES targets were identified from this CMD by selecting probable red giant branch (RGB) stars with $V \lesssim 20.25$. Fig. 1 shows the location of these stars in the LRIS CMD. We used a spectrograph setting that covers the wavelength range 4450$6880 \AA$ with spectral gaps between adjacent orders, a slit width of $1.15^{\prime \prime}$ and a CCD binning of $2 \times 2$ in the spatial and spectral directions. This gives a spectral resolution of $R \approx 34000$. Each programme star was observed for 420-2400 s, depending on its apparent magnitude (see Table 1).

The data were reduced using the $\mathrm{MAKEE}^{2}$ data reduction package. Since our spectra were obtained with the original purpose of studying the internal cluster dynamics (Côté et al. 2002), the exposure times - which were chosen adaptively on the basis of target magnitude - have low signal-to-noise $(S / N)$ ratios.

\footnotetext{
${ }^{2}$ MAKEE was developed by T. A. Barlow specifically for reduction of Keck HIRES data. It is freely available on the World Wide Web at the Keck Observatory home page, http://www2 . keck . hawaii . edu/ inst/hires/makeewww
}

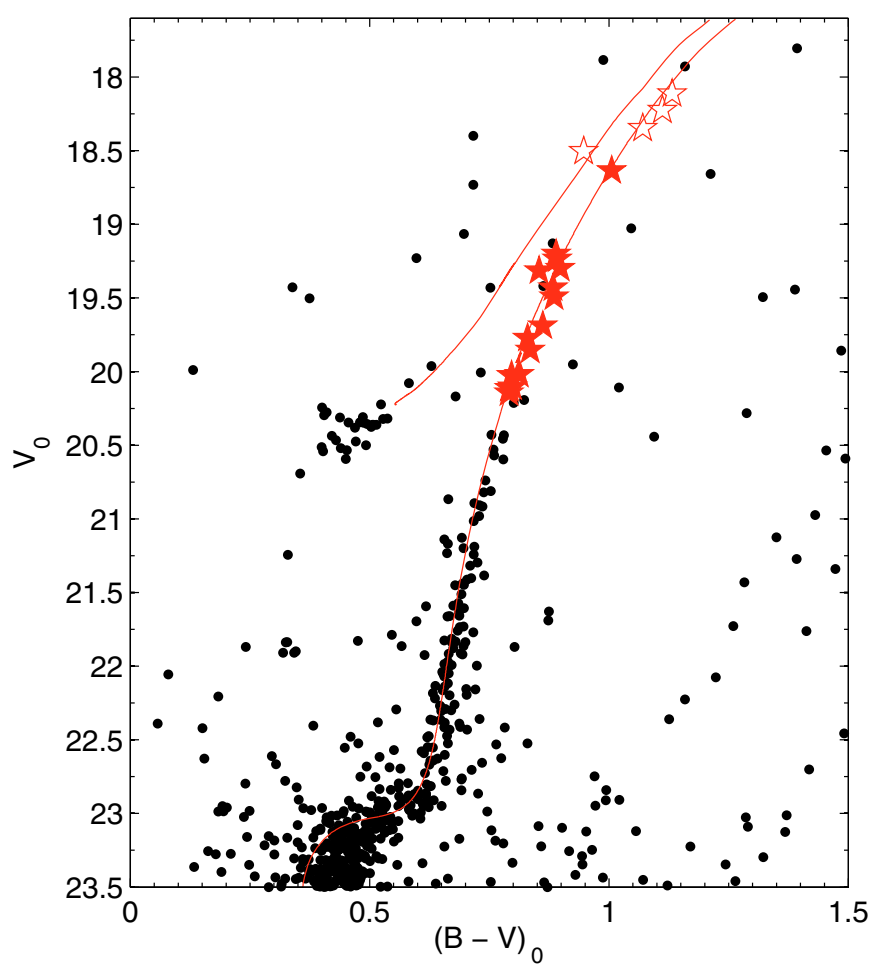

Fig. 1. Colour magnitude diagram of Pal 3 based on our LRIS photometry. Our HIRES (filled symbols) targets and those subsequently observed with MIKE (open symbols) are highlighted as red stars. Also shown is an $\alpha$-enhanced Teramo isochrone (Pietrinferni et al. 2004) with an age of $10 \mathrm{Gyr}$ and an $[\mathrm{Fe} / \mathrm{H}]$ of $-1.6 \mathrm{dex}$, shifted by our best reddening and distance modulus estimates of 0.04 and 19.80 mag.

Thus, the spectra are adequate for the measurement of accurate radial velocities but not for abundance analyses of individual stars. As a result of our observation and reduction strategy, we reach $S / N$ ratios of 4-7 per pixel in the order containing $\mathrm{H} \alpha$. For the present study, we stack the individual spectra to enhance the $S / N$ ratio (Sect. 3.3) and to perform an effective, integrated abundance analysis (see also McWilliam \& Bernstein 2008).

Radial velocities of the individual targets were measured from a cross correlation against a synthetic spectrum of a red giant with stellar parameters representative of the Pal 3 target stars (see also Sect. 3.1), covering the whole wavelength range, but excluding the spectral gaps. We excluded stars deviating by more than $2 \sigma$ from the cluster's mean radial velocity of $\left\langle v_{\mathrm{r}}\right\rangle \approx$ $92 \mathrm{~km} \mathrm{~s}^{-1}$. A detailed account of the dynamics of Pal 3 will be given in a separate paper.

\subsection{MIKE spectra}

Given the large distance of Pal 3 at $(m-M)_{\mathrm{V}, 0}=19.92 \mathrm{mag}$ (91.2 kpc; cf. Stetson et al. 1999; Hilker 2006), the stars on the upper RGB are faint at $V \sim 18.2-18.7$ mag (Fig. 1). However, we have shown in Koch et al. (2008a) that it is possible to target faint RGB stars in distant systems down to $V \sim 19$ in reasonable integration times, with sufficient $S / N$ ratios to investigate chemical abundances. Thus, we chose to observe the four brightest red giants using the Magellan Inamori Kyocera Echelle (MIKE) spectrograph at the 6.5-m Magellan2/Clay Telescope for detailed abundance analyses. These targets were selected from the radial velocity member list based on our Keck run (Sect. 2.1); three of these stars are also included in Hilker (2006), who lists photometrically selected RGB and AGB member stars (Table 1). 
Table 1. Observing log.

\begin{tabular}{|c|c|c|}
\hline Object $^{a}$ & Date & Exposure time [s] \\
\hline \multicolumn{3}{|c|}{ HIRES } \\
\hline Pal3-2(H2) & Feb. 111999 & $1 \times 420$ \\
\hline Pal3-3 (H3) & Feb. 111999 & $1 \times 420$ \\
\hline Pal3-5 (-) & Mar. 101999 & $1 \times 720$ \\
\hline Pal3-6 (H4) & Feb. 111999 & $1 \times 600$ \\
\hline Pal3-8 (H5) & Feb. 121999 & $1 \times 600$ \\
\hline Pal3-15 (H6) & Feb. 111999 & $1 \times 960$ \\
\hline Pal3-16 (H8) & Feb. 121999 & $1 \times 1080$ \\
\hline Pal3-17 (H9) & Feb. 121999 & $1 \times 1200$ \\
\hline Pal3-18 (H10) & Feb. 111999 & $1 \times 1200$ \\
\hline Pal3-21 (-) & Feb. 111999 & $1 \times 1500$ \\
\hline Pal3-22 (H12) & Feb. 121999 & $1 \times 1200$ \\
\hline Pal3-24 (H13) & Feb. 121999 & $1 \times 1500$ \\
\hline Pal3-26 (H14) & Feb. 121999 & $1 \times 1500$ \\
\hline Pal3-29 (H15) & Mar. 101999 & $1 \times 1800$ \\
\hline Pal3-35 (H19) & Feb. 121999 & $1 \times 1800$ \\
\hline Pal3-36 (H16) & Feb. 121999 & $1 \times 1800$ \\
\hline Pal3-38 (H22) & Feb. 121999 & $1 \times 1800$ \\
\hline Pal3-40 (H21) & Mar. 101999 & $1 \times 2400$ \\
\hline Pal3-41 (H23) & Mar. 101999 & $1 \times 2400$ \\
\hline \multicolumn{3}{|c|}{ MIKE } \\
\hline Pal3-2(H2) & Dec. 31 2008, Jan. 012009 & $4 \times 3600$ \\
\hline Pal3-3 (H3) & Jan. 01 2008, Jan. 022009 & $3 \times 3600$ \\
\hline Pal3-5 (-) & Jan. 022008 & $3 \times 3600$ \\
\hline Pal3-6 (H4) & Jan. 032008 & $4 \times 3600$ \\
\hline
\end{tabular}

${ }^{a}$ IDs preceded by "H" are taken from Hilker (2006).

Our data were collected over four nights in January 2009. By using a slit width of $0.7^{\prime \prime}$ and binning the CCD pixels by $2 \times 2$, we obtained a resolving power of $R \sim 34000$. Our data come from the red and blue sides of the instrument, which cover the wavelength range of 3340-9150 $\AA$, although we will primarily use the red wavelength region above $\sim 4900 \AA$ in our analysis. Each star was typically exposed for 3-4 h, which we split into 1 -h exposures to facilitate cosmic ray removal. On average, the seeing was 1 " with individual exposures as high as $1.5^{\prime \prime}$.

The data were processed within the pipeline reduction package of Kelson $(2000,2003)$, which comprises flat field division, order tracing from quartz lamp flats, and wavelength calibration using built-in Th-Ar lamp exposures that were taken immediately following each science exposure. Continuumnormalisation was performed by dividing the extracted spectra by a high-order polynomial fitted to a spectrum of the essentially line-free hot rotating star HR 9098. Our MIKE spectra have $S / N$ ratios of 30-40 per pixel as measured from the peak of the order containing $\mathrm{H} \alpha$.

\section{Abundance analysis}

We begin with an analysis of our high- $S / N$ MIKE spectra. We shall return to the integrated analysis of the co-added HIRES spectra in the next Section.

\subsection{Line list}

We derive chemical element abundances through a standard equivalent $(\mathrm{EW})$ analysis that closely follows the procedures outlined in Koch \& McWilliam (2008) and Koch et al. (2008a,b). We used the 2002 version of the stellar abundance code MOOG (Sneden 1973). The line list for this work is identical to the one we used in Koch et al. (2008a), which, in turn, was assembled from various sources (see Koch et al. 2008a,b; Koch \& McWilliam 2008; and references therein). Transitions for some heavy elements ( $\mathrm{Zr}, \mathrm{La}, \mathrm{Ce}, \mathrm{Dy})$ were supplemented with data from Shetrone et al. (2003); Sadakane et al. (2004) and Yong et al. (2005). Due to the current poorly-determined absolute abundance scale of the heavy elements in Arcturus (particularly the neutron capture elements), we opted to use the laboratory $g f$ values for our lines available in the literature (Koch et al. 2008a,b and references therein) rather than carrying out a differential abundance analysis relative to this reference star (cf. Koch \& McWilliam 2008). The $E W$ s were measured by fitting a Gaussian profile to the absorption lines using IRAF's splot. The final line lists are given in Table 3 for $E W$ s from the MIKE spectra and in Table 4 for $E W$ s from the co-added HIRES spectra. We comment on individual elements and transitions in Sect. 5.

We accounted for the effects of hyperfine structure for the stronger lines of the odd-Z elements Mn I, Cu I, Ba II, La II, and Eu II, using data for the splitting from McWilliam et al. (1995). However, the hyperfine splitting for Sc II, V I, Co I, and Y II was negligible for the weak lines employed in our study and we ignored this effect for these elements. Finally, we placed our abundances on the Solar scale of Asplund et al. (2005), except for iron, for which we adopted $\log \varepsilon_{\odot}(\mathrm{Fe})=7.50$ as an average of the values found in the literature during the past years (see also McWilliam \& Bernstein 2008).

\subsection{Stellar atmospheres}

Throughout our analysis we interpolated the model atmospheres from the updated grid of the Kurucz ${ }^{3}$ one-dimensional 72-layer, plane-parallel, line-blanketed models without convective overshoot and assuming local thermodynamic equilibrium (LTE) for all species. Our models incorporated the new $\alpha$-enhanced opacity distribution functions, AODFNEW (Castelli \& Kurucz $2003)^{4}$. This seems a reasonable choice since the majority of the metal poor Galactic halo GCs and field stars are enhanced in the $\alpha$-elements by $\approx+0.4$ dex, and we would expect Pal 3 to follow this trend (see also Fig. 1; Sects. 4, 5.4).

Photometric temperatures were obtained from the stellar $(V-K)$ colours using the temperature-colour calibrations of Ramírez \& Meléndez (2005) with $K$-band photometry from 2MASS (Cutri et al. 2003). A reddening of $E(B-V)=0.04$ (Stetson et al. 1999; Hilker 2006), the extinction law of Winkler (1997), and an estimated mean metallicity of -1.6 dex from previous photometric studies were also adopted. Given the large uncertainties in the 2MASS K-magnitudes of our faint targets, the resulting $T_{\text {eff }}(V-K)$ estimates have formal uncertainties of $\pm 170 \mathrm{~K}$ on average. In addition, we derived $T_{\text {eff }}$ from our LRIS $B V$ photometry by employing the temperature calibrations of Alonso et al. (1999). In practice, we adopted the $(V-K)$-based values as initial temperatures for our stellar atmospheres for all stars, with one exception: the AGB star Pal3-6 exhibits too red a $(V-K)$ colour (possibly due to an erroneous $K$-band magnitude or an unresolved blend in the 2MASS) that lead to a $T_{\text {eff }}$ lower by $600 \mathrm{~K}$ compared to the $(B-V)$-based value. We then derived spectroscopic temperatures by demanding excitation equilibrium; that is, by requiring there be no trend in the abundance from the Fe I lines with excitation potential. This procedure typically yields $T_{\text {eff }}$ accurate to within $\pm 100 \mathrm{~K}$, based on the range of reasonable slopes. As a result, the spectroscopic temperatures are slightly higher than the photometric values, by $80 \pm 17 \mathrm{~K}$ on

\footnotetext{
3 http://cfaku5. cfa.harvard. edu/grids.html

4 See http://wwwuser. oat.ts.astro.it/castelli.
} 
Table 2. Properties of the targeted member stars.

\begin{tabular}{ccccccc}
\hline \hline ID & $\begin{array}{c}\alpha \\
(\mathrm{J} 2000.0)\end{array}$ & $\begin{array}{c}\delta \\
(\mathrm{J} 2000.0)\end{array}$ & $\begin{array}{c}V_{0} \\
{[\mathrm{mag}]}\end{array}$ & $\begin{array}{c}(B-V)_{0} \\
{[\mathrm{mag}]}\end{array}$ & $\begin{array}{c}(V-K)_{0} \\
{[\mathrm{mag}]}\end{array}$ & $\begin{array}{c}S / N^{a} \\
{\left[\mathrm{pixel}^{-1}\right]}\end{array}$ \\
\hline Pal3-2 & 100531.57 & +000417.0 & 18.11 & 1.13 & 2.73 & $7 / 44$ \\
Pal3-3 & 100537.08 & +000427.9 & 18.22 & 1.11 & 2.57 & $7 / 32$ \\
Pal3-5 & 100530.70 & +000404.2 & 18.35 & 1.07 & 2.52 & $7 / 36$ \\
Pal3-6 & 100529.71 & +000539.2 & 18.50 & 0.95 & 3.47 & $7 / 31$ \\
Pal3-8 & 100531.05 & +000417.3 & 18.64 & 1.01 & 3.45 & 6 \\
Pal3-15 & 100534.63 & +000406.9 & 19.20 & 0.89 & $\ldots$ & 5 \\
Pal3-16 & 100531.84 & +000416.5 & 19.24 & 0.89 & $\ldots$ & 6 \\
Pal3-17 & 100532.87 & +000436.1 & 19.32 & 0.85 & $\ldots$ & 6 \\
Pal3-18 & 100533.19 & +000357.1 & 19.30 & 0.90 & $\ldots$ & 6 \\
Pal3-21 & 100534.04 & +000513.5 & 19.43 & 0.88 & $\ldots$ & 7 \\
Pal3-22 & 100531.12 & +000416.9 & 19.49 & 0.89 & $\ldots$ & 6 \\
Pal3-24 & 100532.91 & +000426.1 & 19.69 & 0.86 & $\ldots$ & 5 \\
Pal3-26 & 100532.59 & +000432.3 & 19.77 & 0.83 & $\ldots$ & 6 \\
Pal3-29 & 100532.73 & +000436.7 & 19.85 & 0.84 & $\ldots$ & 5 \\
Pal3-35 & 100530.25 & +000337.6 & 20.02 & 0.81 & $\ldots$ & 6 \\
Pal3-36 & 100529.92 & +000453.9 & 20.03 & 0.80 & $\ldots$ & 5 \\
Pal3-38 & 100528.79 & +000345.5 & 20.10 & 0.80 & $\ldots$ & 4 \\
Pal3-40 & 100528.83 & +000436.5 & 20.11 & 0.79 & $\ldots$ & 5 \\
Pal3-41 & 100529.97 & +000403.8 & 20.14 & 0.79 & $\ldots$ & 5 \\
\hline
\end{tabular}

${ }^{a}$ The second number for the first 4 stars gives the $S / N$ from the MIKE spectra.

${ }^{b}$ AGB star (Hilker 2006).

Table 3. Linelist for the MIKE spectra.

\begin{tabular}{cccccccc}
\hline \hline Element & $\begin{array}{c}\lambda \\
{[\AA]}\end{array}$ & $\begin{array}{c}\text { E.P. } \\
{[\mathrm{eV}]}\end{array}$ & $\log g f$ & $\begin{array}{c}E W(\mathrm{Pal3}-2) \\
{[\mathrm{m} \AA]}\end{array}$ & $\begin{array}{c}E W(\mathrm{Pal3}-3) \\
{[\mathrm{m} \AA]}\end{array}$ & $\begin{array}{c}E W(\mathrm{Pal3}-5) \\
{[\mathrm{m} \AA]}\end{array}$ & $\begin{array}{c}E W(\mathrm{Pal3}-6) \\
{[\mathrm{m} \AA]}\end{array}$ \\
\hline$[\mathrm{O} \mathrm{I}]$ & 6300.31 & 0.00 & -9.819 & syn & $\ldots$ & syn & syn \\
$\mathrm{Na} \mathrm{I}$ & 8183.26 & 2.10 & 0.230 & 120 & 136 & 114 & 88 \\
$\mathrm{Na} \mathrm{I}$ & 8194.79 & 2.10 & -0.470 & 143 & 160 & 134 & 124 \\
$\mathrm{Mg} \mathrm{I}$ & 5172.70 & 2.71 & -0.390 & 415 & 433 & 431 & 409 \\
$\mathrm{Mg} \mathrm{I}$ & 5528.42 & 4.35 & -0.357 & 160 & 177 & 167 & 167 \\
$\mathrm{Mg}$ I & 5711.09 & 4.33 & -1.728 & 88 & 84 & 86 & 70 \\
\hline
\end{tabular}

Table 3 is available in its entirety in electronic form via the CDS.

Table 4. Linelist for the co-added HIRES spectra.

\begin{tabular}{ccccccc}
\hline \hline Element & $\begin{array}{c}\lambda \\
{[\AA]}\end{array}$ & $\begin{array}{c}\text { E.P. } \\
{[\mathrm{eV}]}\end{array}$ & $\log g f$ & $\begin{array}{c}E W(\mathrm{All}) \\
{[\mathrm{m} \AA]}\end{array}$ & $\begin{array}{c}E W(\mathrm{Pal3-2,3,4,6)} \\
{[\mathrm{m} \AA]}\end{array}$ & $\begin{array}{c}E W(\text { without Pal-2,3,4,6) } \\
{[\mathrm{m} \AA]}\end{array}$ \\
\hline Mg I & 5172.70 & 2.71 & -0.390 & $\ldots$ & 475 & $\ldots$ \\
$\mathrm{Mg} \mathrm{I}$ & 5528.42 & 4.35 & -0.357 & $\ldots$ & 176 & $\ldots$ \\
$\mathrm{Mg}$ I & 5711.09 & 4.33 & -1.728 & 83 & 85 & $\ldots$ \\
$\mathrm{Al} \mathrm{I}$ & 6696.03 & 3.14 & -1.347 & 36 & 40 & $\ldots$ \\
Si I & 5684.48 & 4.95 & -1.650 & $\ldots$ & 34 & 74 \\
Si I & 5708.41 & 4.95 & -1.470 & 63 & 77 & $\ldots 2$ \\
Si I & 5948.55 & 5.08 & -1.230 & 41 & 72 & $\ldots$ \\
Si I & 6142.48 & 5.62 & -0.920 & $\ldots$ & 22 & $\ldots$ \\
Si I & 6155.13 & 5.61 & -0.750 & 28 & $\ldots$ & \\
\hline
\end{tabular}

Table 4 is available in its entirety in electronic form via the CDS.

average. In practice, we adopted the spectroscopic $T_{\text {eff }}$ to enter our atmospheres, as this provides the most reliable and independent determination. In Table 5 we list the atmospheric parameters of the red giants analysed with MIKE.

Photometric gravities were derived from the basic stellar structure equations (e.g., Koch \& McWilliam 2008) using the above temperatures, our $V$-band photometry, and adopting a dereddened distance modulus for Pal 3 of 19.92 mag, which was found to yield a satisfactory fit to our CMD in Fig. 1 (cf. Stetson et al. 1999; Hilker 2006). Comparison of the colours and magnitudes of the Pal 3 red giants against a set of Teramo
Table 5. Atmospheric parameters of the MIKE stars.

\begin{tabular}{|c|c|c|c|c|c|}
\hline \multirow{2}{*}{ ID } & \multicolumn{3}{|c|}{$\bar{T}_{\text {eff }}[\mathrm{K}]$} & \multirow{2}{*}{$\log g$} & \multirow{2}{*}{$\begin{array}{c}\xi \\
{\left[\mathrm{km} \mathrm{s}^{-1}\right]}\end{array}$} \\
\hline & $(B-V)$ & $(V-K)$ & (spec) & & \\
\hline Pal3-2 & 4360 & 4370 & 4470 & 1.08 & 2.10 \\
\hline Pal3-3 & 4390 & 4490 & 4520 & 1.16 & 2.30 \\
\hline Pal3- & 4440 & 4530 & 4630 & 1.28 & 2.20 \\
\hline Pal3-6 & 4610 & 3960 & 4700 & 1.38 & 2.20 \\
\hline
\end{tabular}

isochrones (Pietrinferni et al. 2004) with an age of $10 \mathrm{Gyr}$ and an $[\mathrm{Fe} / \mathrm{H}]$ of $-1.6 \mathrm{dex}$ (Stetson et al. 1999; Hilker 2006) indicates an 
Table 6. Error analysis for the red giants Pal3-2 (RGB) and Pal3-6 (AGB).

\begin{tabular}{|c|c|c|c|c|c|c|c|c|c|}
\hline \multirow{2}{*}{ Ion } & \multicolumn{2}{|c|}{$\Delta T_{\text {eff }}$} & \multicolumn{2}{|c|}{$\Delta \log g$} & \multicolumn{2}{|c|}{$\overline{\Delta \xi}$} & \multicolumn{2}{|c|}{$\overline{\Delta[\mathrm{M} / \mathrm{H}]}$} & \multirow{2}{*}{$\mathrm{ODF}$} \\
\hline & $-100 \mathrm{~K}$ & $+100 \mathrm{~K}$ & $-0.2 \mathrm{dex}$ & $+0.2 \mathrm{dex}$ & $-0.2 \mathrm{~km} \mathrm{~s}^{-1}$ & $+0.2 \mathrm{~km} \mathrm{~s}^{-1}$ & $-0.1 \mathrm{dex}$ & $+0.1 \mathrm{dex}$ & \\
\hline Fe I & -0.13 & 0.14 & 0.01 & $<0.01$ & 0.10 & -0.10 & 0.01 & -0.01 & 0.01 \\
\hline $\mathrm{Fe}$ II & 0.10 & -0.08 & -0.10 & 0.09 & 0.06 & -0.06 & -0.03 & 0.02 & -0.09 \\
\hline $\mathrm{NaI}$ & -0.08 & 0.06 & $<0.01$ & 0.01 & 0.06 & -0.06 & 0.01 & -0.01 & 0.01 \\
\hline Mg I & -0.09 & 0.07 & 0.04 & -0.04 & 0.07 & -0.07 & 0.01 & $<0.01$ & 0.02 \\
\hline Si I & $<0.01$ & $<0.01$ & -0.02 & 0.02 & 0.02 & -0.02 & $<0.01$ & 0.01 & -0.01 \\
\hline K I & -0.16 & 0.14 & $<0.01$ & $<0.01$ & 0.12 & -0.12 & 0.02 & -0.02 & 0.02 \\
\hline $\mathrm{Ca} \mathrm{I}$ & -0.11 & 0.09 & 0.02 & -0.02 & 0.08 & -0.08 & 0.01 & -0.01 & 0.03 \\
\hline Sc II & -0.01 & $<0.01$ & -0.08 & 0.08 & 0.02 & -0.04 & -0.03 & 0.03 & -0.08 \\
\hline Ti I & -0.22 & 0.21 & $<0.01$ & -0.02 & 0.09 & -0.09 & 0.02 & -0.02 & 0.02 \\
\hline Ti II & $<0.01$ & -0.02 & -0.06 & 0.09 & 0.10 & -0.08 & -0.02 & 0.02 & -0.07 \\
\hline V I & -0.18 & 0.18 & 0.02 & -0.02 & 0.02 & -0.01 & 0.01 & $<0.01$ & 0.04 \\
\hline $\mathrm{Cr}$ I & -0.17 & 0.17 & 0.02 & -0.02 & 0.10 & -0.09 & 0.02 & -0.01 & 0.04 \\
\hline Mn I & -0.10 & 0.10 & 0.02 & -0.02 & 0.01 & -0.01 & 0.01 & 000 & 0.03 \\
\hline Co I & -0.13 & 0.14 & $<0.01$ & $<0.01$ & 0.02 & -0.01 & $<0.01$ & $<0.01$ & 0.02 \\
\hline $\mathrm{Ni}$ I & -0.10 & 0.10 & -0.01 & 0.01 & 0.04 & -0.04 & $<0.01$ & $<0.01$ & 0.01 \\
\hline $\mathrm{Cu} \mathrm{I}$ & -0.14 & 0.12 & $<0.01$ & -0.02 & 0.06 & -0.06 & 0.01 & -0.01 & 0.03 \\
\hline Sr II & -0.04 & 0.02 & -0.04 & 0.02 & 0.02 & -0.04 & -0.03 & 0.03 & -0.09 \\
\hline Y II & -0.02 & 0.02 & -0.07 & 0.08 & 0.05 & -0.04 & -0.03 & 0.03 & -0.08 \\
\hline Zr II & -0.02 & $<0.01$ & -0.08 & 0.08 & $<0.01$ & -0.02 & -0.03 & 0.02 & -0.07 \\
\hline Ba II & -0.05 & 0.04 & -0.06 & 0.10 & 0.17 & -0.15 & -0.02 & 0.03 & -0.09 \\
\hline La II & -0.05 & 0.03 & -0.07 & 0.08 & 0.01 & -0.02 & -0.03 & 0.03 & -0.09 \\
\hline Nd II & -0.04 & 0.03 & -0.08 & 0.08 & 0.03 & -0.04 & -0.03 & 0.03 & -0.08 \\
\hline Eu II & -0.01 & $<0.01$ & -0.08 & 0.09 & 0.01 & -0.01 & -0.03 & 0.03 & -0.09 \\
\hline Fe I & -0.14 & 0.13 & 0.01 & -0.01 & 0.09 & -0.08 & 0.01 & -0.01 & 0.04 \\
\hline Fe II & 0.02 & -0.03 & -0.07 & 0.06 & 0.06 & -0.06 & -0.02 & 0.02 & -0.05 \\
\hline $\mathrm{Na} \mathrm{I}$ & -0.07 & 0.06 & 0.01 & -0.01 & 0.01 & -0.06 & 0.01 & -0.01 & 0.03 \\
\hline Mg I & -0.08 & 0.08 & 0.05 & -0.04 & 0.06 & -0.05 & 0.01 & $<0.01$ & 0.02 \\
\hline Si I & -0.04 & 0.02 & $<0.01$ & $<0.01$ & 0.02 & -0.02 & $<0.01$ & $<0.01$ & 0.01 \\
\hline K I & -0.12 & 0.10 & $<0.01$ & -0.02 & 0.08 & -0.06 & 0.02 & -0.01 & 0.04 \\
\hline $\mathrm{Ca} \mathrm{I}$ & -0.09 & 0.08 & 0.02 & -0.01 & 0.05 & -0.05 & 0.01 & $<0.01$ & 0.03 \\
\hline Sc II & -0.01 & $<0.01$ & -0.08 & 0.08 & 0.04 & -0.03 & -0.02 & 0.03 & -0.07 \\
\hline $\mathrm{Ti}$ I & -0.18 & 0.17 & 0.02 & -0.01 & 0.06 & -0.05 & 0.02 & -0.01 & 0.05 \\
\hline Ti II & -0.01 & -0.02 & -0.07 & 0.06 & 0.08 & -0.08 & -0.02 & 0.02 & -0.07 \\
\hline V I & -0.18 & 0.16 & 0.02 & -0.02 & $<0.01$ & -0.02 & 0.01 & -0.01 & 0.04 \\
\hline $\mathrm{Cr}$ I & -0.14 & 0.14 & 0.02 & -0.02 & 0.07 & -0.06 & 0.02 & -0.01 & 0.05 \\
\hline Mn I & -0.11 & 0.10 & 0.02 & $<0.01$ & 0.01 & $<0.01$ & 0.01 & $<0.01$ & 0.03 \\
\hline Co I & -0.15 & 0.15 & 0.01 & $<0.01$ & 0.02 & -0.01 & 0.01 & $<0.01$ & 0.03 \\
\hline $\mathrm{Ni} I$ & -0.12 & 0.11 & $<0.01$ & $<0.01$ & 0.03 & -0.04 & 0.01 & $<0.01$ & 0.03 \\
\hline $\mathrm{Cu} \mathrm{I}$ & -0.14 & 0.14 & $<0.01$ & -0.02 & 0.02 & -0.02 & 0.01 & 0.01 & 0.03 \\
\hline Sr II & -0.04 & 0.04 & -0.02 & 0.04 & 0.04 & -0.04 & -0.02 & 0.03 & -0.07 \\
\hline Y II & -0.02 & 0.02 & -0.08 & 0.06 & 0.06 & -0.04 & -0.02 & -0.02 & -0.06 \\
\hline Ba II & -0.04 & 0.03 & -0.07 & 0.08 & 0.16 & -0.13 & -0.02 & 0.02 & -0.07 \\
\hline La II & -0.04 & 0.04 & -0.06 & 0.08 & 0.08 & -0.02 & -0.02 & -0.02 & -0.06 \\
\hline Nd II & -0.04 & 0.04 & -0.06 & 0.08 & 0.08 & -0.02 & -0.02 & -0.02 & -0.06 \\
\hline Eu II & -0.01 & 0.02 & -0.07 & 0.08 & 0.01 & $<0.01$ & -0.03 & 0.03 & -0.07 \\
\hline
\end{tabular}

average stellar mass of $0.84 M_{\odot}$ for the red giants and $0.78 M_{\odot}$ for the AGB star, which we adopted for the $\log g$ determinations. Uncertainties in the distance and photometry imply an average error in our gravities of 0.12 dex. We note that ionisation equilibrium is not fulfilled in our stars, where we find a mean deviation of the neutral and ionised species of $[\mathrm{Fe} \mathrm{I} / \mathrm{Fe} \mathrm{II}]=$ $-0.07 \pm 0.01 \mathrm{dex}$, while the $[\mathrm{Ti} \mathrm{I} / \mathrm{II}]$ ratio is $0.02 \pm 0.04 \mathrm{dex}$.

We did not attempt to enforce equilibrium by marginal changes in $\log g$ (see also Koch \& McWilliam 2008), but note that an increase of $\log g$ by $0.18 \pm 0.03$ dex would settle the discrepancy at an $[\mathrm{Fe} / \mathrm{H}]$ higher by $0.05 \pm 0.01$ dex on average (see also Sect.4; Table 6). A mild increase of the temperature scale by $37 \pm 3 \mathrm{~K}$ would also reinstall the ionisation balance at marginally higher metallicities.

We then determined microturbulent velocities, $\xi$, from the plot of abundances versus $E W$ of the iron lines, which yields $\xi$, typically to within $0.2 \mathrm{~km} \mathrm{~s}^{-1}$. The resulting microturbulent velocity values of $\sim 2.2 \mathrm{~km} \mathrm{~s}^{-1}$ are somewhat higher than those found in red giants with similar parameters (e.g., Cayrel et al. 2004; Yong et al. 2005). This is likely an artifact of the relatively low $S / N$ ratios of our spectra and the asymmetrical abundance errors of the stronger lines (Magain 1984). However, our analysis reassures us that the high microturbulent velocities from the $E W$ plot are appropriate for our spectra.

Since there is no prior knowledge of the individual stellar metallicities, we initially adopted the cluster mean of -1.6 dex (Hilker 2006, and references therein) as input for the model atmospheres. An independent estimate can be reached from two indicators.

First, our spectra contain the near-infrared calcium triplet (CaT) lines at 8498, 8452, and $8662 \AA$; these lines are a wellcalibrated indicator of the metallicities of red giants in Galactic 
Table 7. Abundance results of the giants targeted with MIKE.

\begin{tabular}{|c|c|c|c|c|c|c|c|c|c|c|c|c|}
\hline \multirow{2}{*}{ Element $^{a}$} & \multicolumn{3}{|c|}{ Pal3-2 } & \multicolumn{3}{|c|}{$\begin{array}{l}\text { Pal3-3 } \\
\end{array}$} & \multicolumn{3}{|c|}{ Pal3-5 } & \multicolumn{3}{|c|}{ Pal3-6 } \\
\hline & {$[\mathrm{X} / \mathrm{Fe}]$} & $\sigma$ & $\bar{N}$ & {$[\mathrm{X} / \mathrm{Fe}]$} & $\sigma$ & $N$ & {$[\mathrm{X} / \mathrm{Fe}]$} & $\sigma$ & $N$ & {$[\mathrm{X} / \mathrm{Fe}]$} & $\sigma$ & $N$ \\
\hline$\overline{\mathrm{Fe}_{\mathrm{CaT}}^{b}}$ & -1.56 & 0.14 & $\ldots$ & -1.58 & 0.14 & $\ldots$ & -1.63 & 0.14 & $\ldots$ & -1.61 & 0.17 & $\ldots$ \\
\hline $\mathrm{Fe}_{\mathrm{MgI}}^{c}$ & -1.51 & 0.13 & $\ldots$ & -1.51 & 0.16 & $\ldots$ & -1.58 & 0.13 & $\ldots$ & -1.67 & 0.14 & $\ldots$ \\
\hline $\mathrm{Fe} \mathrm{I}$ & -1.59 & 0.24 & 141 & -1.54 & 0.27 & 137 & -1.56 & 0.27 & 139 & -1.62 & 0.22 & 121 \\
\hline $\mathrm{Fe}$ II & -1.52 & 0.19 & 9 & -1.50 & 0.16 & 6 & -1.49 & 0.17 & 10 & -1.52 & 0.21 & 8 \\
\hline O I & 0.29 & $\ldots$ & 1 & $\ldots$ & $\ldots$ & .. & 0.30 & $\ldots$ & 1 & 0.32 & .. & 1 \\
\hline $\mathrm{Na}$ & 0.13 & 0.01 & 2 & 0.27 & 0.01 & 2 & 0.13 & 0.02 & 2 & 0.00 & 0.12 & 2 \\
\hline $\operatorname{Mg} I$ & 0.36 & 0.11 & 3 & 0.37 & 0.08 & 3 & 0.47 & 0.04 & 3 & 0.48 & 0.08 & 3 \\
\hline Si I & 0.44 & 0.08 & 5 & 0.46 & 0.10 & 4 & 0.50 & 0.02 & 2 & 0.57 & 0.12 & 2 \\
\hline $\mathrm{K}_{\mathrm{I}}$ & 0.51 & & 1 & 0.48 & & 1 & 0.58 & & 1 & 0.24 & & 1 \\
\hline $\mathrm{Ca} \mathrm{I}$ & 0.31 & 0.12 & 14 & 0.27 & 0.08 & 14 & 0.33 & 0.12 & 14 & 0.30 & 0.14 & 12 \\
\hline Sc II & 0.26 & 0.08 & 5 & 0.40 & 0.07 & 4 & 0.13 & 0.07 & 4 & 0.21 & 0.04 & 6 \\
\hline Ti I & 0.39 & 0.11 & 20 & 0.31 & 0.13 & 8 & 0.37 & 0.12 & 12 & 0.23 & 0.14 & 12 \\
\hline Ti II & 0.33 & 0.11 & 5 & 0.22 & 0.16 & 2 & 0.19 & 0.10 & 4 & 0.22 & 0.23 & 4 \\
\hline V I & 0.13 & 0.07 & 8 & 0.10 & 0.11 & 7 & 0.20 & 0.12 & 4 & 0.10 & $\cdots$ & 1 \\
\hline Cr I & 0.01 & 0.09 & 10 & 0.13 & 0.12 & 8 & 0.15 & 0.15 & 6 & 0.00 & 0.13 & 5 \\
\hline Mn I & -0.32 & 0.07 & 3 & & & & -0.23 & 0.11 & 3 & -0.37 & 0.14 & 3 \\
\hline Co I & 0.11 & 0.05 & 5 & 0.22 & 0.11 & 4 & 0.17 & 0.06 & 3 & 0.15 & 0.06 & 2 \\
\hline Ni I & -0.04 & 0.16 & 24 & 0.05 & 0.19 & 17 & -0.03 & 0.14 & 15 & 0.01 & 0.24 & 17 \\
\hline $\mathrm{Cu} \mathrm{I}$ & -0.26 & . & 1 & -0.25 & & 1 & -0.22 & $\ldots$ & 1 & -0.47 & $\ldots$ & 1 \\
\hline Sr II & -0.46 & 0.00 & 2 & -0.42 & 0.08 & 2 & $\ldots$ & $\ldots$ & $\ldots$ & -0.27 & 0.10 & 2 \\
\hline Y II & -0.03 & 0.04 & 2 & 0.12 & 0.04 & 2 & -0.02 & $\ldots$ & 1 & -0.08 & $\ldots$ & 1 \\
\hline Zr II & 0.03 & $\ldots$ & 1 & 0.32 & $\ldots$ & 1 & 0.13 & $\ldots$ & 1 & $\ldots$ & .. & $\cdots$ \\
\hline Ba II & 0.04 & 0.11 & 3 & -0.02 & 0.19 & 3 & -0.00 & 0.08 & 3 & 0.02 & 0.05 & 3 \\
\hline La II & 0.40 & 0.05 & 3 & 0.36 & $\ldots$ & 1 & 0.36 & .. & 1 & 0.44 & $\ldots$ & 1 \\
\hline Nd II & 0.31 & 0.02 & 2 & 0.31 & $\ldots$ & 1 & 0.31 & 0.08 & 2 & 0.29 & $\ldots$ & 1 \\
\hline Eu II & 0.74 & 0.01 & 2 & 0.61 & 0.11 & 2 & 0.67 & 0.07 & 2 & 0.80 & 0.18 & 2 \\
\hline
\end{tabular}

${ }^{a}$ Ionised species and $\mathrm{O}$ are given relative to Fe II.

${ }^{b}$ Metallicity estimate based on the calcium triplet calibration of Rutledge et al. (1997a,b), on the metallicity scale of Carretta \& Gratton (1997).

${ }^{c}$ Metallicity estimate based on the Mg I calibration of Walker et al. (2007), on the metallicity scale of Carretta \& Gratton (1997).

GCs (Armandroff \& Zinn 1988; Rutledge et al. 1997a,b; Carretta $\&$ Gratton 1997). The measurement of the CaT from highresolution data, however, should be treated with caution since the wings are strong, and any blends with weaker sky residuals, telluric lines, or neutral metal lines will affect their shapes; simplistic line profiles usually fail to fit reliably the CaT lines in very high-resolution spectra. Furthermore, these lines form in the upper chromospheres and are difficult to model reliably (e.g., McWilliam et al. 1995; Battaglia et al. 2008) and setting their continua is not straightforward in our type of spectra. Nonetheless, for a simple order-of-magnitude estimate, we fit the line profiles of the CaT lines as any other absorption line with a Gaussian using splot. The measured EWs were then converted to metallicities on the scale of Carretta \& Gratton (1997) using the calibrations of Rutledge et al. (1997a,b):

$[\mathrm{Fe} / \mathrm{H}]_{\mathrm{CaT}}=-2.66+0.42\left[\Sigma W+0.64\left(V-V_{\mathrm{HB}}\right)\right]$,

where $\Sigma W=0.5 W_{8498}+W_{8452}+0.6 W_{8662}$ is the weighted sum of the $E W \mathrm{~s}$, and $V_{\mathrm{HB}}$ denotes the horizontal branch magnitude, which we adopted as $20.5 \mathrm{mag}$ (Hilker 2006). As a result, we find a mean CaT metallicity $[\mathrm{Fe} / \mathrm{H}]_{\mathrm{CaT}}$ of our stars of $-1.60 \pm$ 0.02 dex, which is in good agreement with both photometric estimates $([\mathrm{Fe} / \mathrm{H}]=-1.57$ dex; Stetson et al. 1999) and the results of low-resolution spectroscopy $\left([\mathrm{Fe} / \mathrm{H}]_{\mathrm{CaT}}=-1.57 \pm 0.19 \mathrm{dex}\right.$; Armandroff et al. 1992), as well as in fair agreement with the value of $-1.70 \pm 0.20$ dex reported by Ortolani \& Gratton (1989).

As a second metallicity indicator, we integrated the $\mathrm{Mg} \mathrm{I}$ lines at $5167,5173 \AA$ to calibrate $[\mathrm{Fe} / \mathrm{H}]$ on the scale of Carretta \& Gratton (1997) as

$[\mathrm{Fe} / \mathrm{H}]_{\mathrm{MgI}}=-2.11+1.76\left[\Sigma \mathrm{Mg}+0.079\left(V-V_{\mathrm{HB}}\right)\right]$.
Here, $\Sigma \mathrm{Mg}=0.547\left(W_{5167}+W_{5173}\right)$ denotes the $\mathrm{Mg}$ index as defined and calibrated in Walker et al. (2007). The respective errors were obtained from the formalism of Cardiel et al. (1998). We find a mean $[\mathrm{Fe} / \mathrm{H}]_{\mathrm{Mg} \text { I }}$ of our stars of $-1.57 \pm 0.04 \mathrm{dex}$, which is in good agreement with the $\mathrm{CaT}$ value. Both the $\mathrm{CaT}$ and $\mathrm{Mg} \mathrm{I}$ values are listed in Table 7, but we emphasise again that these values are meant as initial estimates of the cluster metallicity rather than reliable measures of its abundance scale.

To conclude, we used the abundance of the Fe I lines as input metallicity for the next iteration as we iterated the parameter derivation simultaneously in all parameters until convergence was reached.

\subsection{Co-added HIRES spectra}

Since the HIRES data were originally taken with the sole intention of studying the cluster dynamics (Côté et al. 2002), the $S / N$ ratio of the spectra is low $(\leq 7)$ and does not allow for abundance measurements of the individual stars. Instead, we coadded the spectra to enhance their $S / N$ in order to yield integrated element ratios (cf. McWilliam \& Bernstein 2008). As the highresolution, high- $S / N$ MIKE spectra already resulted in a wealth of abundance information, we followed three procedures so as not to bias the spectral co-addition against these known abundances. First, we co-added all spectra, excluding those stars targeted with MIKE. Second, spectra of all 19 radial velocity members were stacked, including the four MIKE stars. Finally, only the four stars analysed in the previous section were combined into a higher- $S / N$ spectrum. The latter allows us to compare the results of the individual spectra versus the co-added ones and to 


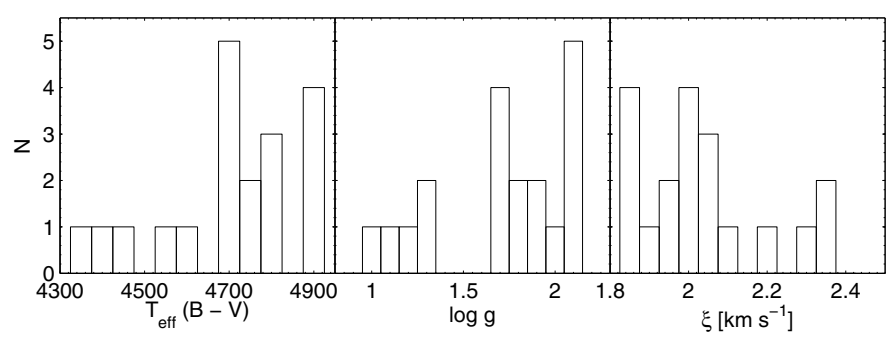

Fig. 2. Distribution of photometric $(B-V)$ temperatures, gravities and microturbulence of the HIRES targets.

look for potential systematic differences between the two methods. Using these three co-adddition schemes, we emulated total $S / N$ ratios of 22,26 , and 14 in the $\mathrm{H} \alpha$-order.

In practice, the spectra were Doppler-shifted and averagecombined after weighting by their individual $S / N$ ratios. In order to yield abundances, we measured the $E W \mathrm{~s}$ from the stacked spectra using the same methods and line lists as in Sect. 3.1 (note, however, the reduced spectral range compared to MIKE). A few of the lines that were measured in the individual MIKE spectra had to be discarded from the co-added line list because they were too weak to measure at the comparatively low $S / N$. Strong lines (such as a few $\mathrm{Mg}$ or Cr lines) for which the coaddition rendered asymmetric profiles and/or line wings too strong to be reliably measured were also excluded. The goal is then to compare these to theoretical $E W$ s from synthetic spectra. To generate these, we computed individual spectra using model atmospheres that represent each star's stellar parameters.

The parameters for our stars were obtained following the procedures described above. Since the targets are relatively faint, they mostly fall below the magnitude limit of the 2MASS (Table 2). Thus, we must rely exclusively on the LRIS $B V$ photometry to obtain $T_{\text {eff. }}$ Surface gravities were derived, as before, from the stars' photometry. Since the parameters of the MIKE stars follow the trend outlined by the metal poor halo stars from Cayrel et al. (2004), we derived microturbulent velocities from a linear fit to their data as $\xi=6.55-9.62 \times 10^{-4} T_{\text {eff }}$. Histograms of the parameters for our HIRES sample are presented in Fig. 2.

The spectral range of our HIRES setup does not contain the near-infrared CaT, but we can obtain metallicity estimates from the $\mathrm{Mg}$ I index (see Eq. (2)). Owing to the low $S / N$ ratios, the uncertainties on the resulting metallicity are inevitably large, typically up to $0.5 \mathrm{dex}$, but we retain this method for an initial, orderof-magnitude estimate. Doing so, we find a mean $[\mathrm{Fe} / \mathrm{H}]_{\mathrm{MgI}}$ of -1.60 dex on the scale of Carretta \& Gratton (1997) with a $1 \sigma$ scatter of 0.28 dex.

With the resulting atmospheres in hand, we computed theoretical $E W \mathrm{~s}$ for the transitions in our line list using MOOG's ewfind driver and combined them into a mean value, $\langle E W\rangle$, using the same weighting scheme as for the observations:

$\langle E W\rangle=\frac{\sum_{i=1}^{N} w_{i} E W_{i}}{\sum_{i=1}^{N} w_{i}}$,

where the weights $w_{i}$ are proportional to the $S / N$ ratios as in the case of co-adding the observed spectra. A comparison of $\langle E W\rangle$ to the observed co-added $E W$ then yields the cluster's integrated abundance ratio for each element. Note that this method presupposes that there is no significant abundance scatter present along the RGB and all stars have the same mean abundances for all chemical elements.

\section{Abundance errors}

The systematic errors on our abundances from MIKE were determined by computing eight new stellar atmospheres, in which each stellar parameter $\left(T_{\mathrm{eff}}, \log g, \xi,[M / \mathrm{H}]\right)$ was varied by its typical uncertainty (as estimated in Sect. 3.1). In addition, we re-ran the analysis using the solar-scaled opacity distributions, ODFNEW, which corresponds to an uncertainty in the input $[\alpha / \mathrm{Fe}]$ ratio of 0.4 dex. With these new atmospheres, new element ratios were determined and we list in Table 6 the abundance differences from those derived using the best-fit atmospheric parameters. This procedure was performed for the stars Pal3-2 and Pal3-6, which cover the full range in $T_{\text {eff }}$.

As a measure for the total systematic uncertainty, we sum in quadrature the contributions from each parameter, although we note that this yields conservative upper limits; the real underlying errors will be smaller due to the covariances of the atmosphere parameters, in particular, that between temperature and gravity (e.g., McWilliam et al. 1995; Johnson 2002; Koch et al. 2008b). As Table 6 shows, the total error on the iron abundances is thus 0.13 dex for the neutral and 0.17 for the ionised species. The $\alpha$ elements are typically uncertain to within 0.15 dex, although there are differences between individual elements (i.e., smaller errors for $\mathrm{Si}$ and $\mathrm{Ca}$, and errors on the $[\mathrm{Ti} / \mathrm{Fe}]$ ratios that are slightly larger). Likewise, the iron peak and neutron capture elements show abundance errors of 0.13-0.17 dex, on average, with the exception of $\mathrm{Ba}$, for which we find a slightly larger uncertainty of $\sim 0.21$ dex. As already noted by Ramírez \& Cohen (2003), the Ti I, V I, and Cr I abundance ratios show a strong dependence on $T_{\text {eff }}$. This is explained by their low excitation potential - at the temperatures of the stars studied here, these species begin to change from fully ionised to fully neutral. Ba II, on the other hand, shows a strong trend with microturbulence due to the generally strong lines. None of the measured abundance ratios is strongly affected by changes in the input metallicties $[M / \mathrm{H}]$, while changes in the $[\alpha / \mathrm{Fe}]$ ratio of the atmospheres lead to a larger impact on all ionised species compared to the neutral stages.

In Table 7 we list the $1 \sigma$ line-to-line scatter and number of lines that was used to derive the abundance ratios listed in this table. This error component yields a measure of the random error accounting for the spectral noise, uncertainties in the atomic parameters, and insufficiencies in the atmosphere models themselves. For elements with many measurable lines, like Fe, Ca, $\mathrm{Ti}$, or $\mathrm{Ni}$, the systematic uncertainties will dominate, whereas for elements with only a few detectable transitions, the line-toline scatter is the dominant error source. For those elements for which only one line was detectable, we adopted an uncertainty of 0.10 dex. For all other elements, we assumed a minimum random error of 0.05 dex (e.g., Ramírez \& Cohen 2003). We will return to the question of intrinsic versus real stellar scatter in Sect. 6.1.

In the case of the co-added HIRES analysis, systematic uncertainties are more difficult to evaluate as the errors on individual stellar parameters propagate through the weighted averaging of the spectra. However, as the $1 \sigma$ line-to-line scatter on those measurements in Table 8 indicates, the statistical error component is large and overwhelms any systematic dependence on the weighted and averaged stellar parameters. This can be due to the low spectral $S / N$, even after the co-addition. In particular, an accurate placement of the continuum is not easily achieved in the stacked low $S / N$ spectra and can lead to random over- and underestimates of measured $E W \mathrm{~s}$. Accordingly, we adopted a minimum random abundance error of 0.10 dex for the HIRES results 
Table 8. Abundance results from the co-added HIRES spectra.

\begin{tabular}{|c|c|c|c|c|c|c|c|c|c|}
\hline \multirow{2}{*}{ Element } & \multicolumn{3}{|c|}{ All } & \multicolumn{3}{|c|}{ Pal3-2,3,4,6 } & \multicolumn{3}{|c|}{ Without Pal3-2,3,4,6 } \\
\hline & {$[\mathrm{X} / \mathrm{Fe}]$} & $\sigma$ & $N$ & {$[\mathrm{X} / \mathrm{Fe}]$} & $\sigma$ & $N$ & {$[\mathrm{X} / \mathrm{Fe}]$} & $\sigma$ & $N$ \\
\hline $\mathrm{Fe}_{\mathrm{MgI}}^{a}$ & -1.58 & 0.18 & $\ldots$ & -1.65 & 0.20 & $\cdots$ & -1.54 & 0.18 & $\cdots$ \\
\hline $\mathrm{Fe} \mathrm{I}$ & -1.52 & 0.43 & 113 & -1.49 & 0.69 & 103 & -1.37 & 0.54 & 105 \\
\hline Fe II & -1.32 & 0.36 & 5 & -1.27 & 0.48 & 3 & -1.22 & 0.35 & 5 \\
\hline Mg I & 0.43 & $\ldots$ & 1 & 0.24 & 0.03 & 3 & $\ldots$ & $\ldots$ & . \\
\hline $\mathrm{Al}$ I & 0.68 & $\ldots$ & 1 & 0.56 & $\ldots$ & 1 & $\ldots$ & $\ldots$ & \\
\hline Si I & 0.34 & 0.28 & 3 & 0.40 & 0.29 & 4 & 0.30 & 0.37 & 3 \\
\hline $\mathrm{Ca} \mathrm{I}$ & 0.38 & 0.20 & 13 & 0.31 & 0.35 & 11 & 0.29 & 0.20 & 16 \\
\hline Sc II & 0.26 & 0.33 & 4 & 0.16 & 0.20 & 4 & 0.22 & 0.14 & 4 \\
\hline Ti I & 0.24 & 0.32 & 18 & 0.13 & 0.18 & 11 & 0.26 & 0.40 & 13 \\
\hline Ti II & 0.35 & 0.32 & 9 & 0.31 & 0.46 & 4 & 0.30 & 0.21 & 6 \\
\hline V I & 0.14 & 0.23 & 4 & 0.15 & 0.22 & 3 & 0.13 & 0.08 & 3 \\
\hline CrI & -0.02 & 0.46 & 7 & -0.19 & 0.44 & 7 & -0.03 & 0.37 & 8 \\
\hline Mn I & -0.38 & 0.02 & 2 & -0.30 & ‥ & 1 & -0.44 & $\ldots$ & 1 \\
\hline Co I & 0.19 & 0.26 & 4 & 0.04 & 0.28 & 4 & 0.00 & 0.01 & 2 \\
\hline Ni I & 0.00 & 0.35 & 9 & -0.13 & 0.18 & 6 & 0.06 & 0.17 & 9 \\
\hline $\mathrm{Cu} \mathrm{I}$ & -0.41 & 0.19 & 2 & -0.62 & $\ldots$ & 1 & -0.40 & 0.13 & 2 \\
\hline Y II & 0.00 & 0.15 & 3 & $\cdots$ & $\ldots$ & .. & 0.00 & 0.20 & 3 \\
\hline Zr II & 0.13 & $\ldots$ & 1 & 0.11 & $\ldots$ & 1 & 0.24 & ․ & 1 \\
\hline Ba II & -0.02 & $\ldots$ & 1 & -0.03 & $\ldots$ & 1 & -0.04 & $\ldots$ & 1 \\
\hline La II & 0.33 & 0.10 & 3 & 0.24 & $\ldots$ & 1 & $\ldots$ & $\ldots$ & 1 \\
\hline Ce II & 0.31 & $\ldots$ & 1 & 0.29 & $\ldots$ & 1 & 0.13 & $\ldots$ & 1 \\
\hline $\mathrm{Nd}$ II & 0.34 & $\ldots$ & 1 & 0.30 & $\ldots$ & 1 & 0.30 & $\ldots$ & 1 \\
\hline Dy II & 0.56 & $\ldots$ & 1 & $\ldots$ & $\ldots$ & $\ldots$ & 0.87 & $\ldots$ & 1 \\
\hline
\end{tabular}

${ }^{a}$ Metallicity estimate based on the Mg I calibration of Walker et al. (2007), on the metallicity scale of Carretta \& Gratton (1997).

and assign an uncertainty of 0.15 dex if only one line could be measured.

\section{Abundance results}

Table 7 lists the abundance ratios relative to Fe I, except for all ionised species and O I from the forbidden line, which we give relative to Fe II. These are also illustrated in the boxplots of Fig. 3 which show the median and interquartile ranges for each element's abundance ratio.

In analogy, Table 8 lists the abundance ratios derived from the co-added HIRES spectra. These result are broadly consistent with those derived from the individual MIKE spectra to within the uncertainties, as also indicated by the red error bars in Fig. 3, which depict the mean $[\mathrm{X} / \mathrm{Fe}]$ and $1 \sigma$ spread obtained from coadding all HIRES spectra. As Fig. 4 shows, the results are invariant against the sample used for co-addition. In the following plots, we will include the data point from co-adding all HIRES spectra, but will focus the discussion and statistics of the Pal 3 abundances from the more accurate MIKE data.

In Fig. 5 we show the run of the $[X / \mathrm{Fe}]$ abundance ratios with effective temperature exemplary for the $\alpha$-elements $\mathrm{O}$ through Ti. All abundance ratios, with the possible exception of $\mathrm{Mg}$ and $\mathrm{Si}$ (see also Sects. 5.4 and 6.1) are consistent with showing no trend with evolutionary stage in that they are constant with respect to $T_{\text {eff }}$ to within the uncertainties. We now briefly comment on our findings for the groups of individual elements.

\subsection{Iron abundance}

From the four red giants with MIKE spectra we find a cluster mean $[\mathrm{Fe} / \mathrm{H}]$ from the neutral lines of $-1.58 \pm 0.02$ (stat.) \pm 0.13 (sys.) dex. This is fully consistent with the CaT based mean metallicity of $-1.60 \pm 0.02$ dex derived above. In particular, we find a mean discrepancy of the CaT and Fe I values of our four stars of only $-0.02 \pm 0.02$ dex. In fact, both our CaT estimate and the more accurate $[\mathrm{Fe} / \mathrm{H}]$ abundance ratio are in excellent agreement with the estimates of Stetson et al. (1999) from CMD fitting and the CaT measurement of Armandroff et al. (1998) ${ }^{5}$. Our value is also marginally consistent with the range of metallicities given by Ortolani \& Gratton (1989) and Hilker (2006) to within the uncertainties. Analysis of the co-added HIRES spectra yields a slightly higher value of $-1.52 \pm 0.04$, which nevertheless agrees with the value from the accurate MIKE analysis to within the uncertainties.

We do not find any trend of $[\mathrm{Fe} / \mathrm{H}]$ with $T_{\text {eff }}$, which is a good indicator of stellar evolutionary status. This reassures us that coadding individual spectra will yield abundance results that are representative of the entire cluster (recall from Fig. 2 that the HIRES targets span $\sim 600 \mathrm{~K}$ across the RGB). Moreover, there is no discernible trend of a deviation of Fe I and II with $T_{\text {eff }}$, so that LTE is a valid approximation at the temperatures and metallicities of our stars (see also Thévenin \& Idiart 1999; Ramírez \& Cohen 2003; Yong et al. 2005).

On the other hand, the ionised iron abundances derived from the co-added HIRES spectra are systematically higher than the neutral values, with a mean $[\mathrm{Fe}$ I/ Fe II] of $-0.19 \pm 0.02$. Within the combined error bars, this discrepancy is significant at $1.4 \sigma$ on average. Typical EWs of the ionised lines range from 30$120 \mathrm{~mA}$. One might argue that non-LTE effects start to affect our stars more strongly the further down we move on the RGB, so that the co-added spectra suffer from the integrated non-LTE corrections. On the other hand, a large deviation of -0.22 dex is already present when only the four brightest spectra (Pal3-2 through Pal3-6) are combined (see middle part of Table 8). Moreover, in their integrated light abundance study of 47 Tuc, McWilliam \& Bernstein (2008) find a small discrepancy

\footnotetext{
5 Note, however, that these authors placed their measurements on the GC abundance scale of Zinn \& West (1984), which yields [Fe/H] larger by up to 0.3 dex in this metallicity regime.
} 

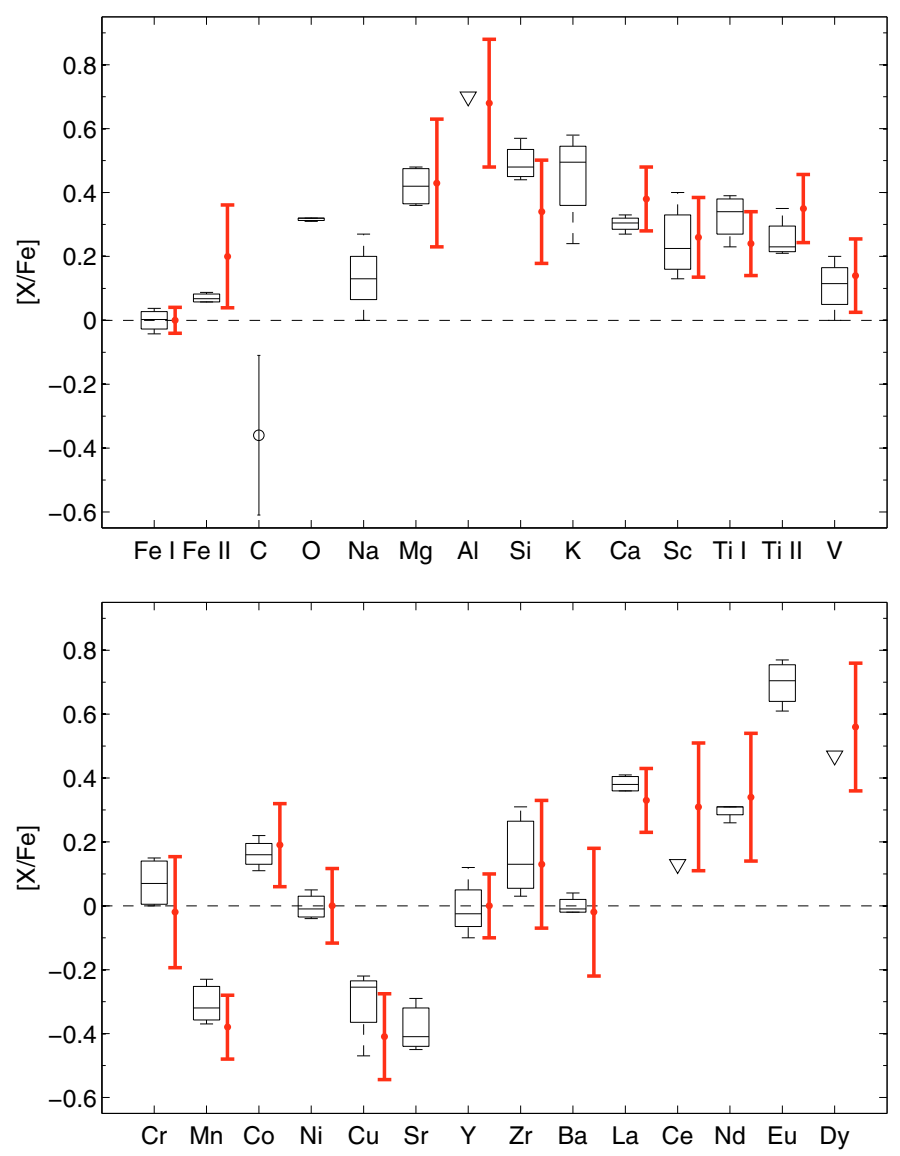

Fig. 3. Boxplots of Pal 3 abundances from the MIKE spectra, relative to $\mathrm{Fe} \mathrm{I}$, for $\mathrm{C}$ through $\mathrm{V}$ (top) and $\mathrm{Cr}$ through Dy (bottom). The boxes designate the mean values and interquartile ranges. Iron abundances are shown relative to the cluster mean. The red offset error bars indicate the mean and $1 \sigma$ scatter derived from the co-added HIRES spectra, while triangles indicate the elements for which upper limits could be derived from co-added MIKE spectra only.

between Fe I and Fe II of 0.03 dex that is well within the respective uncertainties. Whatever the cause, we conclude that Fe II lines seem in general not suitable for establishing a population's iron abundance from a low $S / N$ spectral co-addition as employed in the present work (cf. Kraft \& Ivans 2003).

\section{2. $C, O$ abundances}

Although the low $S / N$ ratios of the individual blue spectra did not allow us to resolve the $\mathrm{CH}$ G-band at $4323 \AA$, we derived a constraint of the mean $[\mathrm{C} / \mathrm{Fe}]$ abundance ratio by co-adding the four MIKE spectra. We then fit synthetic spectra with stellar parameters that provided a representative mean of the actual MIKE targets (Table 2) by varying carbon abundances in a leastsquares sense to the stacked spectrum. For this purpose, we employed a $\mathrm{CH}$ line list with isotope splitting, assuming a ${ }^{12} \mathrm{C} /{ }^{13} \mathrm{C}$ ratio of 10 , as typically found in evolved giants, and $g f$-values by B. Plez (A. Frebel, private communication; see Frebel et al. 2007). As Fig. 10 implies, the co-added spectrum is reasonably well fit with a $[\mathrm{C} / \mathrm{Fe}]$ ratio of $\sim-0.36 \pm 0.25 \mathrm{dex}$, which we state as an order of magnitude estimate of Pal 3's overall carbon abundance. Given the luminosities of our targets (on the upper RGB; $\left.\log \left(L / L_{\odot}\right) \sim 2.7\right)$, the $[\mathrm{C} / \mathrm{Fe}]$ ratio we find is fully representative of evolved stars that had typical standard carbon
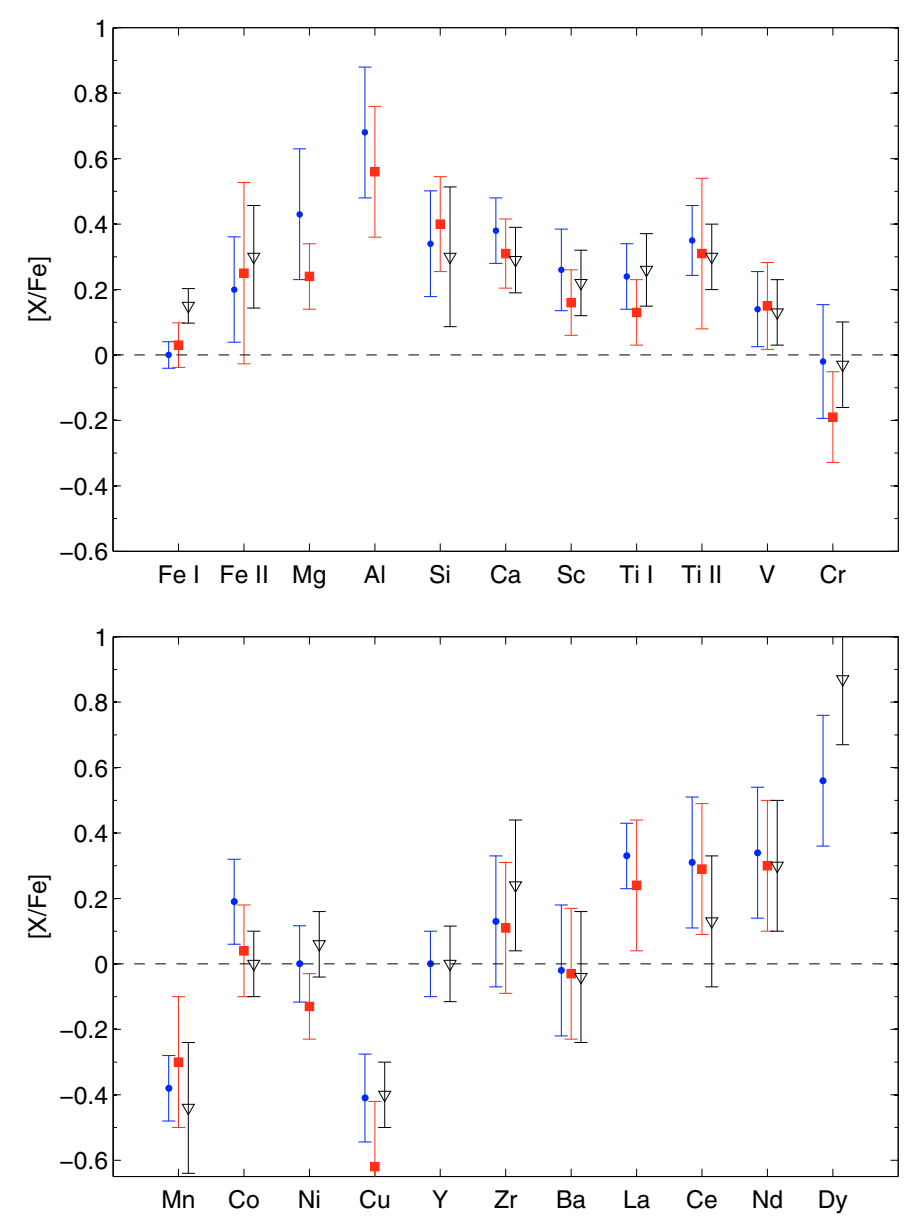

Fig. 4. Elemental abundances derived from co-added HIRES spectra, for $\mathrm{O}$ through $\mathrm{Cr}$ (top) and $\mathrm{Mn}$ through Dy (bottom), including different samples of stars: all HIRES stars (blue circles), Pal3-2,3,4 and 6 only (red squares), and all stars but excluding the latter (black triangles).

abundances when they formed, and which were depleted to the observed level in the course of their stellar evolution (Gratton et al. 2004; Aoki et al. 2007; see also Frebel et al. 2009; their Fig. 11).

Oxygen abundances could not be directly inferred from $E W$ measurements of the weak [OI] 6300, $6363 \AA$ lines, as these are heavily affected by sky emission lines. Instead, we synthesized the spectral region around the $6300 \AA$ line and varied the $[\mathrm{O} / \mathrm{Fe}]$ abundance to achieve an acceptable fit to the portion of the stellar line that is unaffected by telluric [O I] in the line wing. Since this procedure is still hampered by a low $S / N$ around the absorption feature, we assigned a minimum error bar of 0.2 dex to the [O/Fe] values. For Pal3-3, the emission residual directly coincides with the stellar line and no $[\mathrm{O} / \mathrm{Fe}]$ could be derived for this star. As a result, we find a mild $[\mathrm{O} / \mathrm{Fe}]$ enhancement in our stars of typically 0.3 dex.

\subsection{Light odd-Z elements: $\mathrm{Na}, \mathrm{Al}, \mathrm{K}$}

The Na-D 5889, $5895 \AA$ lines, which are present in our MIKE spectra, generally yield abundance results that differ systematically from those derived from the near-infrared set of lines at 8183, 8194 A (e.g., Ivans et al. 2001; Ramírez \& Cohen 2003). Since the former resonance lines are too strong at the metallicities in question and strongly affected by telluric absorption to 


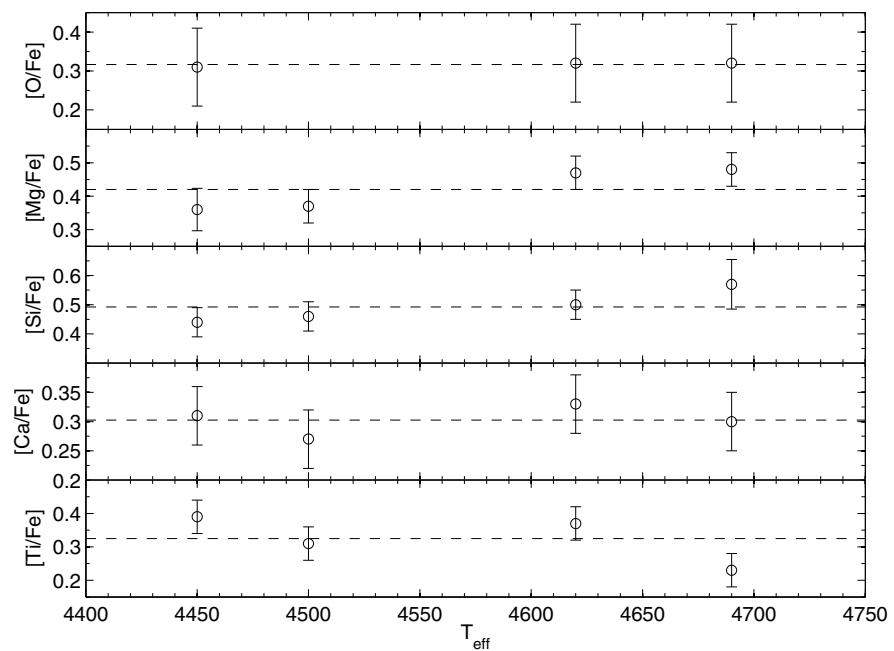

Fig. 5. Abundance ratios for $\mathrm{O}, \mathrm{Mg}, \mathrm{Si}, \mathrm{Ca}$ and $\mathrm{Ti}$ as a function of stellar effective temperature. Shown as dashed lines are the cluster mean values. Error bars are $1 \sigma$ random errors, where a minimum error was assumed for elements with only one measurable line.

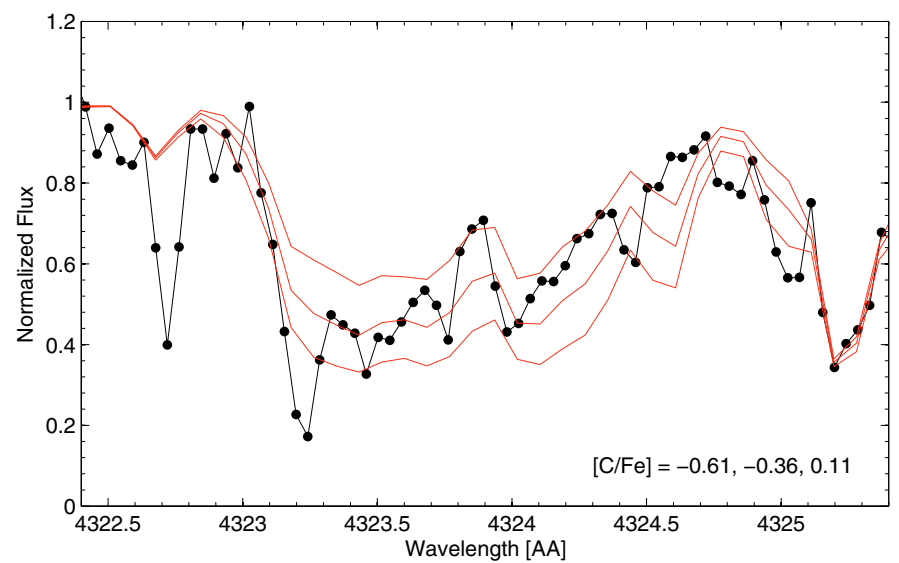

Fig. 6. Co-added MIKE spectra around the $\mathrm{CH}$ feature at $4323 \AA$. Shown as red lines are the best fit synthetic spectrum and those with $[\mathrm{C} / \mathrm{Fe}]$ differing by \pm 0.25 dex.

be measured reliably, we chose to measure the sodium abundance ratios from the $8193,8194 \AA$ doublet instead of averaging the full set of the four deviant lines. Unfortunately, no $\mathrm{Na}$ abundances could be inferred from the HIRES spectra, since the $\mathrm{Na}-\mathrm{D}$ falls on the spectral gaps and the near-infrared lines are not covered by our set-up. For stars with similar atmosphere parameters to our Pal 3 stars, the calculations of Takeda et al. (2003) show that downward non-LTE corrections become more severe with increasing $E W$ for the $\mathrm{Na}$ I lines at 8183 and $8195 \AA$. At those relatively large $E W \mathrm{~s}$ of $\sim 140 \mathrm{~m} \AA$ in our stars, the Takeda et al. (2003) results suggest abundance corrections that reduce our LTE values by $0.45-0.50$, if non-LTE effects on Fe are ignored. This would bring the sodium abundances in accord with the slightly depleted Galactic halo stars (Fig. 7). Given the complexity of the non-LTE, we proceed by adopting the LTE abundance ratios for further discussions and list those in Table 7. Since we only have $[\mathrm{O} / \mathrm{Fe}]$ abundances available for three of our stars, it is not fully representative to test our limited sample for any anti-correlations between $\mathrm{Na}$ and $\mathrm{O}$ within $\mathrm{Pal} 3$ itself. The $[\mathrm{O} / \mathrm{Fe}]$ ratio in our stars is approximately constant at $0.30 \mathrm{dex}$, but we note that the nominal $[\mathrm{Na} / \mathrm{O}]$ ratios of our stars are consistent with the trends outlined by the numerous samples

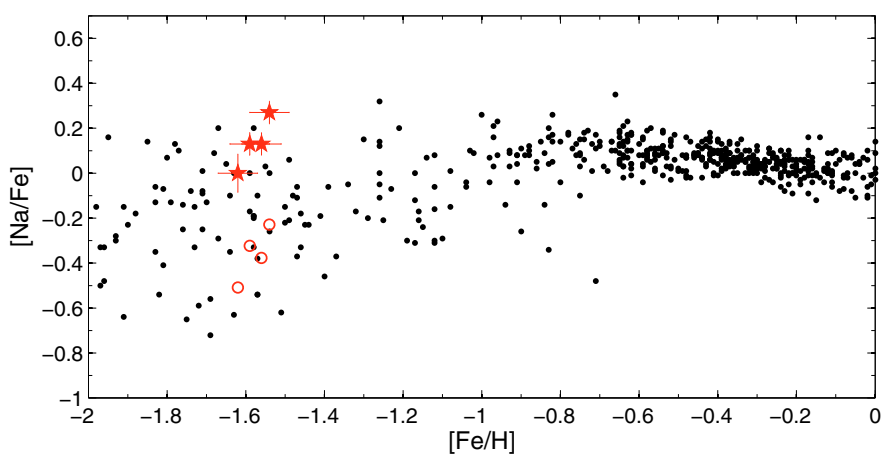

Fig. 7. Sodium abundance ratios for the Pal 3 stars (red symbols) and the Galactic halo and disks (black dots; see text for references). The star symbols show our derived LTE abundance ratios, while open circles illustrate the abundances after applying the Na non-LTE corrections from Takeda et al. (2003) for stars at the same metallicities as Pal 3.

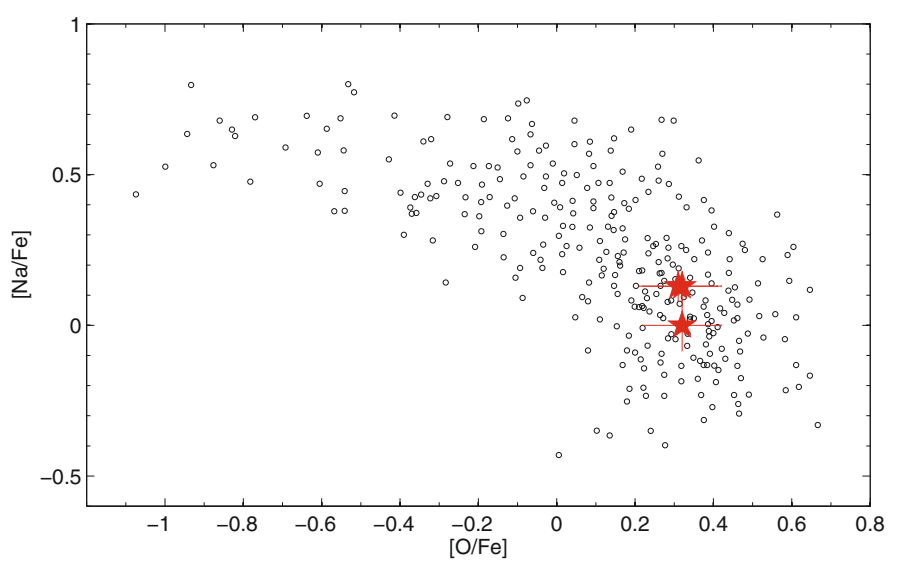

Fig. 8. Na-O Anti-correlation for Galactic GC stars (small black circles) after Gratton et al. (2004) and Carretta (2006). Our Pal 3 measurements are shown as red stars.

of Galactic GC stars (Fig. 8; Gratton et al. 2004; Carretta 2006). The present data can neither confirm nor refute the Na-O anticorrelation in Pal 3.

Unfortunately, no aluminum line could be detected in any of our MIKE spectra. However, we derived upper limits from marginal detections of the $6696 \AA$ line in the co-added HIRES spectra. Thus, we find an $[\mathrm{Al} / \mathrm{Fe}]$ of $\lesssim 0.7 \mathrm{dex}$, which is in accord with the values found in GCs of comparable metallicities (e.g., Cohen \& Meléndez 2005a).

The determination of potassium abundances in red giants is a delicate venture for two reasons. First, the two strongest resonance lines at 7698, $7664 \AA$ fall into a window of strong telluric contamination. Second, the $[\mathrm{K} / \mathrm{Fe}]$ ratio is strongly affected by non-LTE effects. Regarding the first issue, we are fortunate that the $7698 \AA$ resonance line in Pal 3 is sufficiently free from telluric absorption at the GC's radial velocity. With respect to the departure from LTE, Takeda et al. (2009) suggest that downward non-LTE corrections in mildly metal poor GC giants can be as high as $\sim-0.35$ dex (see also Zhang et al. 2006). At the metallicity of M5 ( - 1.5 dex), Takeda et al. (2009) predict NLTE corrections of the order of -0.2 dex, albeit for cooler giants than in our sample. As for the case of sodium, an accurate treatment of the departure from LTE in our stars is clearly beyond the scope of this paper, but we note that an extrapolation of the Takeda et al. (2009) corrections to the $T_{\text {eff }}$ of our targets, would result in our K-abundances being lowered by $0.4-0.6$ dex. 


\subsection{The $\alpha$-elements: $\mathrm{Mg}, \mathrm{Si}, \mathrm{Ca}, \mathrm{Ti}$}

$\mathrm{Mg}$ and $\mathrm{Si}$ are the only elements for which we find an indication of increasing abundances with increasing $T_{\text {eff }}$ of the order of $(0.7 \pm 0.3) \operatorname{dex}(1000 \mathrm{~K})^{-1}$. It should be noted, however, that the $\mathrm{Si}$ abundance is generally based on 2-5 weak lines that are often affected by low $S / N$ and, at their high excitation potential, may be prone to temperature uncertainties.

Likewise, the Ti II abundance in Pal3-3 was derived from only two lines, since the other transitions were too strongly affected by noise or blends. All in all, the $[\alpha / \mathrm{Fe}]$ abundance ratios of $\mathrm{Mg}, \mathrm{Si}, \mathrm{Ca}$, and $\mathrm{Ti}$ are enhanced to about $0.4 \mathrm{dex}$, which is the canonical value found in Galactic halo field stars and GCs over a broad range of metallicities (see also Sect. 6; Fig. 10) and Galactocentric radii.

\subsection{Iron peak elements: $\mathrm{Sc}, \mathrm{V}, \mathrm{Cr}, \mathrm{Mn}, \mathrm{Co}, \mathrm{Ni}, \mathrm{Cu}$}

In all our stars the even-Z iron-peak element ratios $[\mathrm{Cr} / \mathrm{Fe}]$ and $[\mathrm{Ni} / \mathrm{Fe}]$ follow the abundance of iron so that $[\mathrm{X} / \mathrm{Fe}] \sim 0$. This trend is representative of the values found in the halo population and other GCs (e.g., Yong et al. 2005). Nissen \& Schuster (1997) reported a significant correlation of their $[\mathrm{Ni} / \mathrm{Fe}]$ abundance ratios in Galactic disk and halo stars with $[\mathrm{Na} / \mathrm{Fe}]$, which was subsequently confirmed for other stellar systems such as the dSphs (e.g., Shetrone et al. 2003). As for the Na-O anti-correlation, our sample of four stars is too small to investigate any such intrinsic correlation in Pal 3, but we note that our values agree well with the overall $[\mathrm{Ni} / \mathrm{Na}]$ relation defined by Galactic stars.

For Sc, V, and $\mathrm{Co}$, we find ratios that are mildly enhanced to $\sim 0.2$ dex and thus slightly larger than the Solar values found in the other clusters (e.g., Yong et al. 2005; Cohen \& Meléndez 2005 a), which may be an artifact of the usually low $S / N$ around the few weak absorption lines in question, while $\mathrm{Cr}$ and $\mathrm{Ni}$ have more and better defined transitions in our spectra. The odd-Z elements $\mathrm{Mn}$ and $\mathrm{Cu}$ are depleted with respect to iron; their mean values of $-0.31([\mathrm{Mn} / \mathrm{Fe}])$ and $-0.30([\mathrm{Cu} / \mathrm{Fe}])$ are consistent with the element ratios found in a number of Galactic GCs and the moderately metal poor halo field stars, albeit falling towards the higher end of the envelope (Cayrel et al. 2004; McWilliam et al. 2003; McWilliam \& Smecker-Hane 2005). We note, however, that $[\mathrm{Cu} / \mathrm{Fe}]$ has been derived from the $5105 \AA$ line only, which may be affected by blends that lead to larger uncertainties.

\subsection{Neutron-capture elements: Sr, Y, Zr, Ba, La, Nd, Eu (Ce, Dy)}

Although lying in the blue, low- $S / N$ part of our MIKE spectra, we estimated [Sr/Fe] from the $4077,4215 \AA$ resonance lines that, with $E W$ s of 200-250 m $\AA$, are strong in our stars, yet reach consistent abundances. This yields depleted Sr abundance ratios of typically -0.3 dex. The abundances of the n-capture elements Y, La and Nd were determined from typically 2-3 sufficiently strong lines, while the $[\mathrm{Zr} / \mathrm{Fe}]$ ratio is solely based on the weak $5112 \AA$ line. [Eu/Fe], on the other hand, was measured from the weak ( 20-30 m $\AA$ ) 6437 and $6645 \AA$ lines. As a result, we find slightly supersolar $\mathrm{Y}$ and $[\mathrm{Zr} / \mathrm{Fe}]$ abundance ratios, whilst the elements heavier than $\mathrm{La}$ are enhanced to $[\mathrm{X} / \mathrm{Fe}]$ of $0.35 \mathrm{dex}$ up to $0.8 \mathrm{dex}$ for $[\mathrm{Eu} / \mathrm{Fe}]$, which we also confirmed from spectral synthesis and measurements in a higher $S / N$ coadded MIKE spectrum. The $[\mathrm{Ba} / \mathrm{Fe}]$ ratios, derived from the 5853, 6141, $6496 \AA$ lines, are found to be Solar and are therefore compatible with the $[\mathrm{Ba} / \mathrm{Fe}]$ ratios of Galactic halo stars above $[\mathrm{Fe} / \mathrm{H}] \gtrsim-2$ dex. We note, however, that this may be an artifact
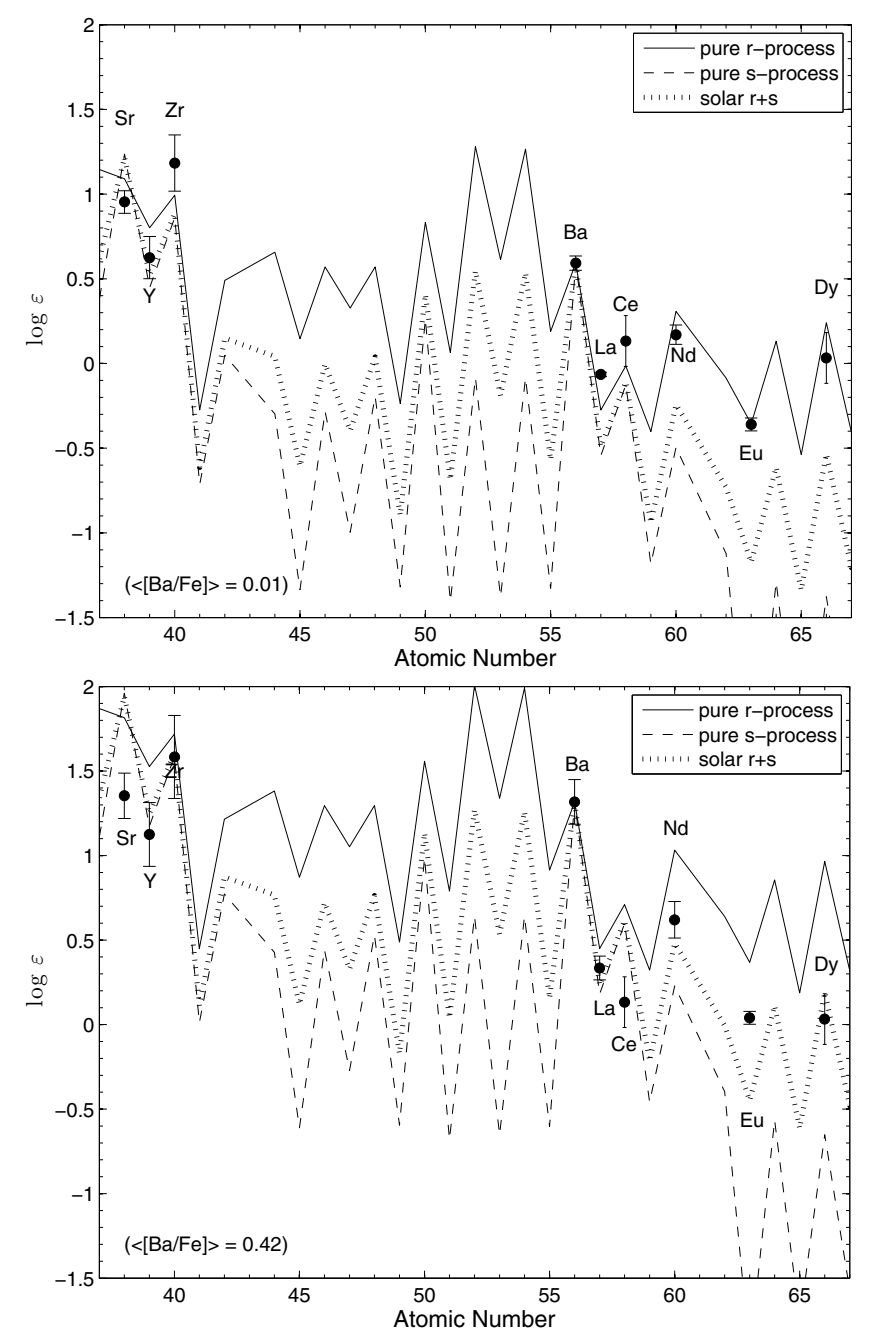

Fig. 9. Mean neutron-capture abundance ratios in comparison to the scaled-solar r- and s-process ratios of Burris et al. (2000), each normalised to $\mathrm{Ba}$. The bottom panel assumes a lower microturbulence of $\xi=1.7 \mathrm{~km} \mathrm{~s}^{-1}$ (cf. Table 4 ), leading to $[\mathrm{Ba} / \mathrm{Fe}]$ ratios higher by $\sim 0.4$ dex. The error bars each indicate the $\sigma$-spreads.

of the high microturbulent velocities adopted for our stars. While most transitions for other elements are relatively unaffected by this parameter, the Ba lines are very sensitive to changes in $\xi$. For example, if we were to assume a lower microturbulence of $\sim 1.7 \mathrm{~km} \mathrm{~s}^{-1}$ as suggested by a canonical $T_{\text {eff- }}$ or $\log g-\xi$ relation (e.g., Cayrel et al. 2004; Yong et al. 2005), our stars would have higher $[\mathrm{Ba} / \mathrm{Fe}]$ abundance ratios with a mean of 0.42 dex. As the adopted values for $\xi$ yield a consistent $E W$ equilibrium, we proceed by using the higher values, but urge caution in the interpretation of our measured $[\mathrm{Ba} / \mathrm{Fe}]$.

Although neither Ce nor Dy could be measured from the blue, low- $S / N$ part of our individual MIKE spectra, we derived upper limits from a co-added spectrum of the four stars (as in Sect. 3.2), therefore yielding an estimate of the GC's mean abundance ratios for these elements. This procedure succeeded for the weak Ce 5274.2 $\AA$ and Dy $5169.8 \AA$ lines, from which we derived a [Ce II /Fe II] ([Dy II /Fe II]) of $0.13(0.47)$ dex, respectively. The transition probabilities for these were taken from Sadakane et al. (2004; and references therein) and we based the error on the resulting $[\mathrm{Ce}, \mathrm{Dy} / \mathrm{Fe}]$ ratios on the acceptable range in measured $E W \mathrm{~s}$, which led to a conservative estimate of $\sim \pm 0.15$ dex. In Fig. 9, we compare our observed abundance 
ratios for the neutron-capture elements $(Z \geq 38)$ to the Solarscaled abundance patterns (Burris et al. 2000), where we normalised the curves to the same $\mathrm{Ba}$ abundance, both for the values obtained for the high microturbulence of our stars (top panel) and for a lower value of $1.7 \mathrm{~km} \mathrm{~s}^{-1}$ (bottom panel). In the top panel (higher $\xi$ ), all the heavy elements save Y are fully consistent with the solar $r$-process abundance curve, which implies that these stars formed out of material that was not considerably enriched by the AGB stars that produce the $s$-process elements, but predominantly by the short-lived supernovae ( $\mathrm{SNe}$ ) of type II. Given the only moderately low iron abundance of Pal 3 and the younger age of this cluster, this may seem surprising as the long-lived AGB stars could have formed and evolved in the GC's early phases to contribute s-process elements (cf. Sadakane et al. 2004). If Pal 3's heavy elements are confirmed to be r-process dominated, this would be the second known example of a GC with such abundance patterns after M $15([\mathrm{Fe} / \mathrm{H}]=-2.3 \mathrm{dex}$; Sneden et al. 2000). Given the possibility of a lower microturbulence, the bottom panel suggests, however, that a higher $[\mathrm{Ba} / \mathrm{Fe}]$ and therefore a standard $r+s$ mixture is not ruled out by our data. An $r$-process dominance is further underscored by the relatively high $[\mathrm{Eu} / \mathrm{Fe}]$ in conjunction with the resulting low $[s / r]$ ratios at the metallicity of this GC (Fig. 16); note that the unsaturated Eu and La lines are relatively unscathed with respect to the adopted microturbulence. We can further investigate the question of the $r$-process origin of the heavy elements in Pal 3 by differentiating the production channels into the weak $r$-process component $(Z<56)$, which may occur in massive SNe II with progenitor masses above $20 M_{\odot}$ (e.g., Wanajo \& Ishimaru 2006), and the main $r$-process (all $Z \geq 38$; in 8-10 $M_{\odot}$ SNeII; Qian \& Wasserburg 2003). Our [Ba/Sr] ratio of $0.35-0.50$ dex is consistent with the values found in halo stars at the same metallicity and is indicative of the main $r$-process without any need to invoke an enrichment by very massive stars in Pal 3 as found in low-mass environments such as the ultra-faint dSph galaxies (Koch et al. 2008a; Frebel et al. 2009).

\section{Discussion}

\section{1. (No) Abundance variations}

As noted above, it is impossible to draw firm conclusions about chemical abundance variations using a low $S / N$ sample of only four stars. Nonetheless, to get an order-of-magnitude estimate, we followed Cohen \& Melendez (2005a) in defining the spread ratio, $\mathrm{SR}$, as the $1 \sigma$ abundance spread divided by the total systematic and random error (added in quadrature) of each element. Thus the SR is an indication of whether the observed spread is a real intrinsic star-to-star scatter or a mere statistical fluctuation. In addition, we computed the probability, $P$, that the corresponding (observed) $\chi^{2}$ will be exceeded by chance. We find SRs ranging from 0.05 to a maximum of 1.05 for Na. These values are close to, or well below, unity so that the observed abundances spreads are likely caused by the measurement errors only. Furthermore, none of the elements has a $P\left(\chi^{2}\right)$ smaller than $0.5 \%$, which Cohen \& Melendez (2005a) define as a threshold for bonafide abundance variations.

Again, the lowest value is found for $\mathrm{Na}$, with $P\left(\chi^{2}\right)=40 \%$, while all other elements show no evidence of any variation with probabilities much in excess of $60 \%$. While one would expect the scatter of $[\mathrm{Na} / \mathrm{Fe}]$ to be accompanied by a comparable spread in $[\mathrm{O} / \mathrm{Fe}]$, we find no evidence of any significant oxygen variation. We note, however, that the SR of 0.05 for $[\mathrm{O} / \mathrm{Fe}]$ is only

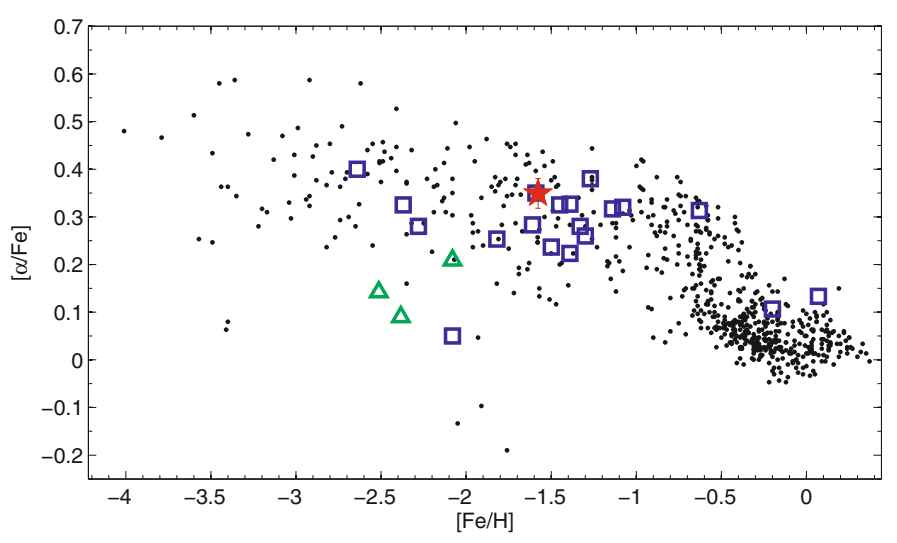

Fig. 10. Same as Fig. 7, but for the mean $[\alpha / \mathrm{Fe}]$ abundance ratio. Here, the red star symbol denotes the mean and $1 \sigma$-spread of Pal 3 derived from our MIKE spectra. Also indicated as green triangles are mean values for the GCs in the Fornax dSph (Letarte et al. 2006). Blue squares are GC data from the literature (see text for references).

based on three stars and derived from crude spectral synthesis rather than the $E W$ analysis as for the other elements. In this context, the (Kendall rank) probability that $\mathrm{Na}$ and $\mathrm{O}$ in our stars are uncorrelated within the random errors is $(77 \pm 32) \%$, but again we note the potential bias of a low number statistics coupled with different measurement methods of these two elements. Given the close resemblance of our abundance data with stars in other GCs (Fig. 8; Sect. 6.2), we conclude that Pal 3 probably follows the usual abundance variations and anti-correlations caused by internal mixing processes in the giant stars (e.g., Ivans et al. 2001; Gratton et al. 2004), even if the present, sparse data cannot confirm nor rule out any stronger trend. Essentially the same result holds for the $\mathrm{Na}-\mathrm{Ni}$ correlation (at a probability of $70 \pm 28 \%$ ) found in Galactic field and GC stars, with which the Pal 3 abundance ratios are broadly consistent.

The SRs of $[\mathrm{Mg} / \mathrm{Fe}]$ and $[\mathrm{Si} / \mathrm{Fe}]$ are 0.52 and 0.76 , which confirms that neither of these elements shows any significant variations. This also suggests that their marginal trend with $T_{\text {eff }}$ (Fig. 5) is not a real feature. The smallest range of abundances is found for the heavy elements $\mathrm{Ba}, \mathrm{La}$, and $\mathrm{Nd}$ with $\mathrm{P}\left(\chi^{2}\right)$ of 99.3-99.6\%, which is in accord with the findings in other GCs (e.g., Yong et al. 2005), and for Ca at a comparably high level. All in all, we conclude that our limited data are compatible with no star-to-star scatter. A detailed evaluation of the marginal evidence of the canonical abundance (anti-) correlations $(\mathrm{Na} / \mathrm{O}$, $\mathrm{Na} / \mathrm{Ni}$ ) within Pal 3 must await more data. Such a homogeneity in the elements supports the possibility of abundance analyses from co-added spectra of individual stars without the introduction of scatter due to star-to-star variations in the final GC spectrum.

Furthermore, this characteristic clearly rules out Pal 3 being a low-luminosity dwarf galaxy. All such systems studied to date (e.g., Shetrone et al. 2001, 2003; Kirby et al. 2008; Koch 2009) show large abundance spreads of the order of several tenths of a dex.

\subsection{Comparison with other GCs}

As mentioned in the previous Sections, the chemical abundance distributions of Pal 3 show few surprises. All of the elements studied in this work are fully consistent with those ratios found in GCs across a wide range of metallicity and Galactocentric radii. We illustrate this behavior in Figs. 10-12, in which we 


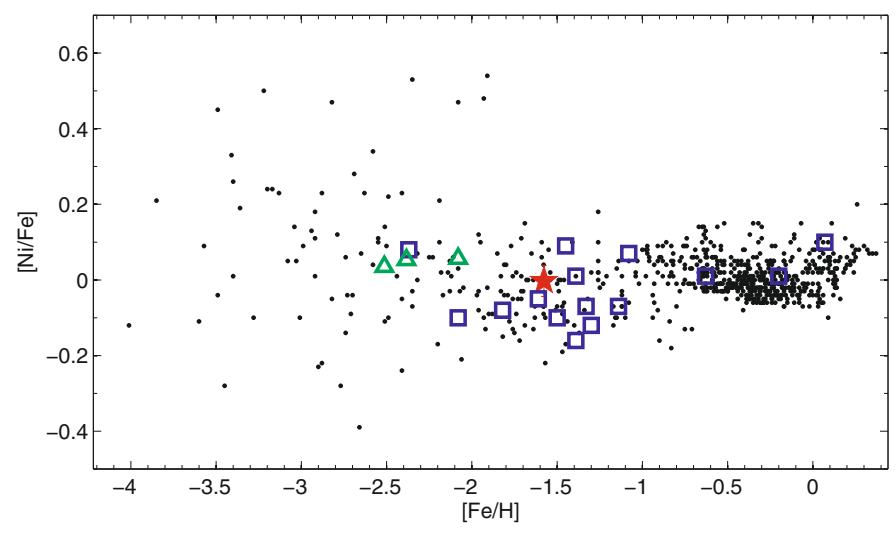

Fig. 11. Same as Fig. 10, but for the $[\mathrm{Ni} / \mathrm{Fe}]$ abundance ratio.

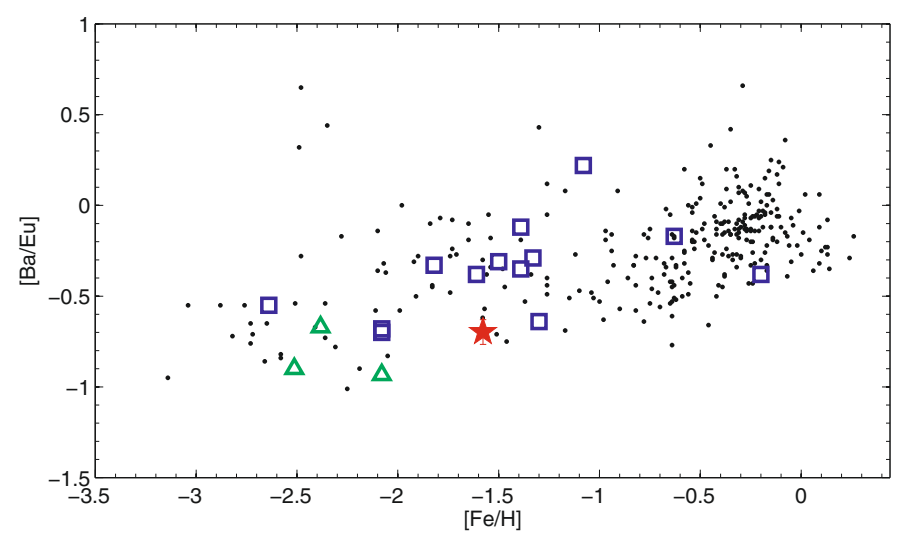

Fig. 12. Same as Fig. 10, but for the $[s / r]$ abundance ratio $[\mathrm{Ba} / \mathrm{Eu}]$.

show the run of the mean $[\alpha / \mathrm{Fe}]^{6},[\mathrm{Ni} / \mathrm{Fe}]$ and $[\mathrm{Ba} / \mathrm{Eu}]$, exemplary for the range of abundance ratios analysed in this work. In analogy with Gratton et al. (2004); Pritzl et al. (2005); Cohen \& Meléndez (2005a); Yong et al. (2005); Geisler et al. (2007) and Koch (2009), we overplot in Figs. 7, 10, 11, 12 our measured abundance ratios on a large number of Galactic thin-disk, thickdisk, and halo stars from the studies of Gratton \& Sneden (1988, 1994); Edvardsson (1993); McWilliam et al. (1995); Ryan et al. (1996); Nissen \& Schuster (1997); McWilliam (1998); Hanson et al. (1998); Burris et al. (2000); Prochaska et al. (2000); Fulbright (2000, 2002); Stephens \& Boesgaard (2002); Johnson (2002); Bensby et al. (2003); Ivans et al. (2003), and Reddy et al. (2003) (see also Pritzl et al. 2005; Geisler et al. 2007; Koch 2009). We further highlight as large open squares a sample of mean GC abundances for M 3 (Cohen \& Meléndez 2005a); M 4 (Ivans et al. 1999); M 5 (Ramírez \& Cohen 2003); M 10 (Haynes et al. 2008); M 13 (Cohen \& Meléndez 2005a); M 15 (Preston et al. 2006); M 71 (Ramírez \& Cohen 2003); M 92 (Shetrone 1996; Sneden et al. 2000); NGC 288 (Shetrone \& Keane 2000); NGC 362 (Shetrone \& Keane 2000); NGC 1851 (Yong \& Grundahl 2008); NGC 2419 (Shetrone et al. 2001); NGC 2808 (Carretta 2006); NGC 3201 (Gonzalez \& Wallerstein 1998); NGC 5694 (Lee et al. 2006); NGC 6397 (James et al. 2004); NGC 6528 (Carretta et al. 2001); NGC 6553 (Carretta et al. 2001; Alves-Brito et al. 2006); NGC 6752

\footnotetext{
${ }^{6}$ Although this average of $[(\mathrm{Mg}+\mathrm{Ca}+\mathrm{Ti}) / 3 \mathrm{Fe}]$ is a convenient and illustrative measure, one should keep in mind that $\mathrm{Mg}, \mathrm{Ca}$, and $\mathrm{Ti}$ are produced in different channels of the SNe II event, therefore limiting somewhat this simplistic mean; see discussions in Venn et al. (2004) and Koch et al. (2008b).
}

(Yong et al. 2005); NGC 7006 (Kraft et al. 1998), and NGC 7492 (Cohen \& Meléndez 2005b). For these plots, no efforts were taken to homogenise the abundance data from the various sources with respect to different approaches in the analysis (i.e., regarding $\log g f$ values and stellar atmospheres), but we did correct for differences in the adopted Solar abundance scales where necessary (see also Cohen \& Meléndez 2005b).

As discussed in Sect. 5, the abundance ratios found in Pal 3 are fully compatible with the trends found in the Galactic halo at comparable metallicities. This holds for the individual $\alpha$ elements as well as the iron peak (Fig. 11) and the majority of the heavy, n-capture elements. The $[\mathrm{Ba} / \mathrm{Eu}]$ ratio (Fig. 12) in the Pal 3 stars is slightly lower than in the halo stars and the GCs at $[\mathrm{Fe} / \mathrm{H}] \sim-1.5 \mathrm{dex}$, but it is still consistent with these components to within the measured uncertainties. We note the following interesting cases:

(1) NGC $5694([\mathrm{Fe} / \mathrm{H}]=-2.08$ dex; Lee et al. 2006): This $\mathrm{GC}$ is peculiar because of a strong deficiency in the $\alpha$-elements by about $0.3-0.4$ dex compared to the bulk of Galactic $\mathrm{GCs}^{7}$. Although the heavy element patterns are different from those found in the dSph galaxies (e.g., Shetrone et al. 2001, 2003; Geisler et al. 2007; Koch 2009, and references therein), such an $\alpha$-depletion, coupled with the large Galactocentric distance of $\sim 30 \mathrm{kpc}$ and its large negative radial velocity, prompted Lee et al. (2006) to conclude that this outer halo cluster is likely of extragalactic origin. As Fig. 10 indicates, the $[\alpha / \mathrm{Fe}]$ pattern of Pal 3 bears no resemblance with that of NGC 5694, while their heavy element ratios (Figs. 11, 12) are comparable to within the uncertainties.

(2) Fornax GCs $(\langle[\mathrm{Fe} / \mathrm{H}]\rangle=-2.33$ dex; Letarte et al. 2006): The Fornax dSph is the only MW dSph satellite known to harbour its own GC system apart from the disrupted Sgr system. Its abundance ratios are compatible with those of the dSph field stars, albeit at fairly low metallicities. In particular, the Fornax GC stars show the canonical depletion in $[\alpha / \mathrm{Fe}]$ with respect to the Galactic halo and are therefore compatible with the ratios of the dSph-like GC NGC 5694 described above. Their [Ba/Eu] ratios are not unlike the value we measured in Pal 3, but, again, representative of the lower metallicity regime around -2.5 to -2.0 dex. In this comparison, Pal 3 is again dissimilar to the dSph populations.

(3) NGC $7492([\mathrm{Fe} / \mathrm{H}]=-1.82 \mathrm{dex}$; Cohen \& Meléndez 2005b): Despite its large Galactocentric distance of $25 \mathrm{kpc}$, this outer halo cluster shows abundance ratios that are fully consistent with GCs of the inner halo such as M3 and M13 at around $10 \mathrm{kpc}$. The intriguing fact that all the common chemical elements studied in M 3, M 13 and NGC 7492 are so similar led Cohen \& Meléndez (2005b) to conclude that, if these clusters were typical representatives of the inner and outer halos, then these components underwent chemical enrichment histories that were indistinguishable. We therefore compare in Fig. 13 (right panel) our measured abundance ratios to those derived by Cohen $\&$ Meléndez (2005b). The error bars include $1 \sigma$ random errors from both studies, added in quadrature. To reach a fair comparison, we accounted for the different $[\mathrm{Fe} / \mathrm{H}]$ and Solar abundance scales used in the abundance analyses, but we did not correct for any potential offsets due to variations in the line list or stellar atmospheres. From this we infer that $42 \%$ (63\%) of the abundance ratios that were measured in common between Pal 3 and NGC 7492 differ by less than $0.10(0.15)$ dex. Accounting for measurement uncertainties, this means that $47 \%(74 \%)$ of the

\footnotetext{
7 Note, however, that the respective abundances in this GC were derived from a single red giant.
} 


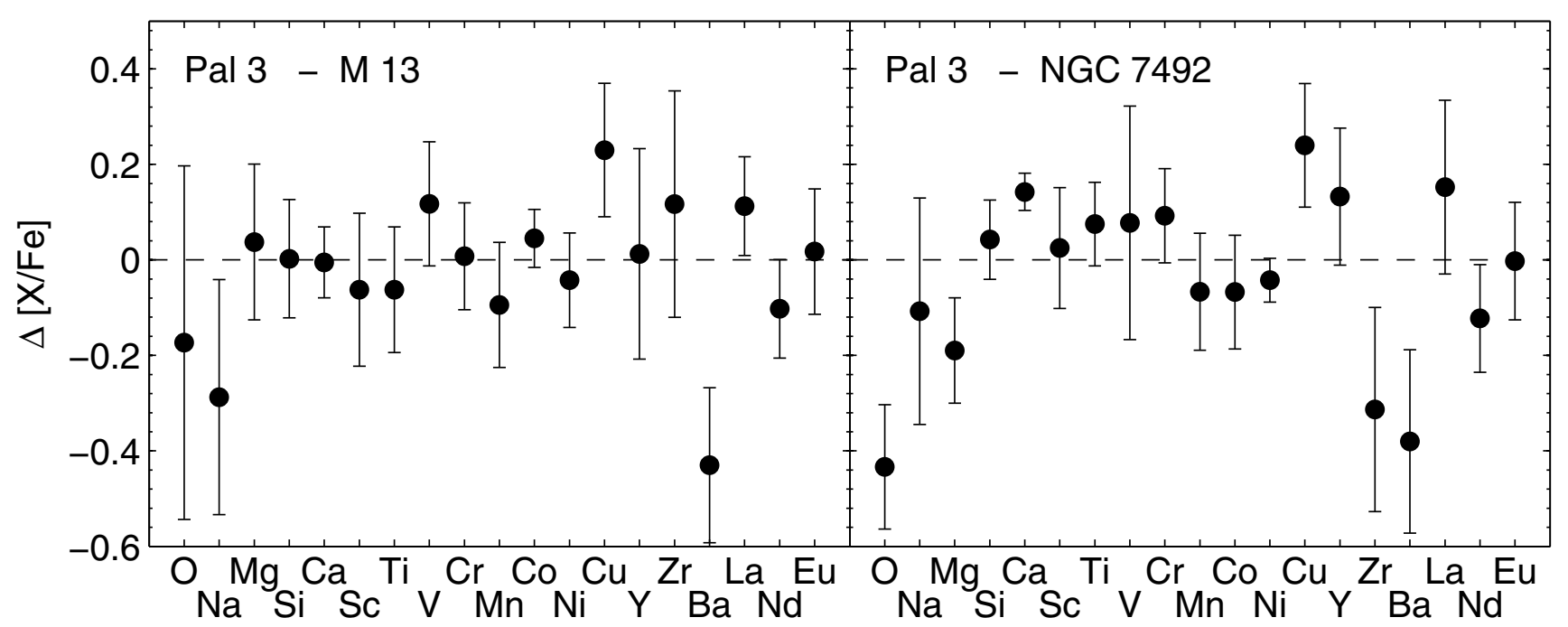

Fig. 13. Abundance differences in the sense $[\mathrm{X} / \mathrm{Fe}]_{\mathrm{Pal} 3}-[\mathrm{X} / \mathrm{Fe}]_{\mathrm{GC}}$ for the inner halo $\mathrm{GC} \mathrm{M} 13\left(R_{\mathrm{GC}}=12 \mathrm{kpc} ;[\mathrm{Fe} / \mathrm{H}]=-1.50 \mathrm{dex} ; \mathrm{Cohen} \&\right.$ Meléndez 2005a) and the outer halo cluster NGC $7492\left(R_{\mathrm{GC}}=25 \mathrm{kpc} ;[\mathrm{Fe} / \mathrm{H}]=-1.82\right.$ dex; Cohen \& Meléndez 2005b). We corrected for different Solar abundance scales. Error bars include the $1 \sigma$-spreads for both Pal 3 and the GCs from literature.

ratios agree to within $1 \sigma(2 \sigma)$, where the largest discrepancies occur for $\mathrm{O}, \mathrm{Zr}$, and $\mathrm{Ba}$. Hence, Pal 3 can probably be considered representative of the outer halo GC population in terms of its chemical abundance ratios.

(4) $M 3, M 13([\mathrm{Fe} / \mathrm{H}]=-1.39,-1.50 \mathrm{dex} ;$ Cohen \& Meléndez 2005a): These clusters have long been considered as archetypical GCs and show overall abundance patterns that are in very good agreement with other moderately metal poor GCs and Galactic halo field stars, which suggests a common evolutionary history (see Cohen \& Meléndez 2005a, for a comprehensive discussion). We show in Fig. 17 (left panel) the analogous comparison of the M13 literature abundance distribution with our Pal 3 measurements, only correcting for $[\mathrm{Fe} / \mathrm{H}]$ and the Solar abundances. The mean metallicities of these GCs differ by a mere 0.08 dex so that any resemblance in the chemical elements would indicate a common chemical history. In fact, $63 \%$ (79\%) of the M13 and Pal 3 abundances measured in common agree to within $0.10(0.15)$ dex; that is, $79 \%(95 \%)$ of the chemical elements agree within better than $1 \sigma(2 \sigma)$. This strengthens the close connection between the inner Galactic halo and the outermost regions, as represented by $\mathrm{Pal} 3$. While the coevolution of the GCs in between $\sim 10$ and $30 \mathrm{kpc}$ (as established by Cohen \& Meléndez 2005a) already poses an important constraint on the common history of the inner and outer halos, the extension of this similarity to the outermost halo at $\sim 100 \mathrm{kpc}$ suggests that the mechanisms of GC formation (excluding those that may originate in $\mathrm{dSph}$ accretion events) may be invariant over the full extent of the MW halo.

Following this argument, an $r$-process dominance of the Pal 3 stars would advocate a similar dominance to be found in these inner halo clusters. As Fig. 17 implies, this is not the case, since the abundance difference between $\mathrm{Pal} 3$ and either of the GCs is in fact largest (by $\sim 0.4 \mathrm{dex}$ ) for $[\mathrm{Ba} / \mathrm{Fe}]$. The heavy element and $[s / r]$ patterns in M3 and M13 found by Cohen \& Meléndez (2005a) are compatible with a regular Solar $r+s$ mix.

\section{Summary}

We have performed a comprehensive abundance analysis of the remote halo GC Pal 3. The fact that our co-added high-resolution, low- $S / N$ spectra yield results consistent with individual, higher $S / N$ spectra is an important step towards future analyses of faint and remote systems, for which no abundance information can yet be gleaned. Although systematic uncertainties and the low $S / N$ ratios complicate such studies, an accuracy of 0.2 dex on most abundance ratios yields information sufficient to place such systems in context with both the inner and outer halo GCs, and the faint dSph galaxies. We were unable to detect significant abundance variations in this GC, with the possible exception of sodium. Our Pal 3 sample is hampered by a small number of stars; within the current data it cannot be ruled out that also this $\mathrm{GC}$ follows the global $(\mathrm{Na} / \mathrm{O}, \mathrm{Na} / \mathrm{Ni})$ correlations defined by Galactic GC stars (Gratton et al. 2004). This clearly contrasts the large abundance spreads found in all dSphs studied to date and argues against Pal 3 being an accreted system.

We find tentative evidence that the heavy elements in Pal 3 are dominated by $r$-process nucleosynthesis, which has to date only been found in very metal poor halo field stars and the more metal poor GC M 15. This statement, however, hinges on the adopted value of the microturbulence. Further studies from higher $S / N$ spectra are clearly desirable to resolve this issue. If real, this finding would pose strong constraints on the cluster's early evolution and pollution phases (e.g, Bekki et al. 2007; Marcolini et al. 2009) and give insight into the relevant gas expulsion time scales and the cluster environment (e.g., Baumgardt et al. 2005; Baumgardt \& Kroupa 2007; Parmentier \& Fritze 2009).

Stetson et al. (1999) noted that the "age difference [between Pal 3, M 3 and M 5] could be smaller if either $[\mathrm{Fe} / \mathrm{H}]$ or $[\alpha / \mathrm{Fe}]$ for the outer halo clusters is significantly lower than ... assumed". Their CMD fitting suggested an $[\mathrm{Fe} / \mathrm{H}]$ of $-1.57 \mathrm{dex}$, assuming $[\alpha / \mathrm{Fe}]=+0.3$ dex. Here we have shown that both the iron and $\alpha$-element abundances have nearly these exact values, rendering it likely that $\mathrm{Pal} 3$ is younger by up to $\approx 2 \mathrm{Gyr}$ than the inner halo reference clusters of comparable metallicity. Despite such an age difference, which led to early notions of the existence of two separate GC populations (e.g., Searle \& Zinn 1978), we have found that Pal 3 is remarkably typical in its abundance patterns, which are almost identical to those in Galactic halo field stars and other, inner (i.e., $R_{\mathrm{GC}}<15 \mathrm{kpc}$ ) and outer $\left(R_{\mathrm{GC}}>25 \mathrm{kpc}\right)$ GCs. While many authors have 
established a metallicity dichotomy between the inner and outer halo field stars, such a strong division does not appear to extend to the chemical abundances of the MW GC system. In short, while an extragalactic origin of the metal-poor stellar halo, e.g., in systems resembling the ultra-faint dSphs (Simon \& Geha 2007; Frebel et al. 2009; Koch 2009) cannot be excluded at present, our observations would appear to rule out such an accretion origin for Pal 3.

Acknowledgements. We are grateful to Anna Frebel for help with the $\mathrm{CH}$ line list. A.K. acknowledges support by an STFC postdoctoral fellowship.

\section{References}

Allen, C., Moreno, E., \& Pichardo, B. 2006, ApJ, 652, 1150

Alonso, A., Arribas, S., \& Martínez-Roger, C. 1999, A\&AS, 140, 261

Alves-Brito, A., Barbuy, B., Zoccali, M., et al. 2006, A\&A, 460, 269

Aoki, W., Beers, T. C., Christlieb, N., et al. 2007, ApJ, 655, 492

Armandroff, T. E., \& Zinn, R. 1988, AJ, 96, 92

Armandroff, T. E., Da Costa, G. S., \& Zinn, R. 1992, AJ, 104, 164

Asplund, M., Grevesse, N., \& Sauval, A. J. 2005, in Cosmic Abundances as

Records of Stellar Evolution and Nucleosynthesis, ed. T. G. Barnes III \& F. N. Bash (San Francisco: ASP), ASP Conf. Ser., 236, 25

Battaglia, G., Irwin, M., Tolstoy, E., et al. 2008, MNRAS, 383, 183

Baumgardt, H., \& Kroupa, P. 2007, MNRAS, 380, 1589

Baumgardt, H., Grebel, E. K., \& Kroupa, P. 2005, MNRAS, 359, L1

Bekki, K., Campbell, S. W., Lattanzio, J. C., \& Norris, J. E. 2007, MNRAS, 377, 335

Bellazzini, M., Ferraro, F. R., \& Ibata, R. 2003, AJ, 125, 188

Belokurov, V., Zucker, D. B., Evans, N. W., et al. 2007, ApJ, 654, 897

Bensby, T., Feltzing, S., \& Lundström, I. 2003, A\&A, 410, 527

Burris, D. L., Pilachowski, C. A., Armandroff, T. E., et al. 2000, ApJ, 544, 302

Cardiel, N., Gorgas, J., Cenarro, J., \& Gonzalez, J. J. 1998, A\&AS, 127, 597

Carollo, D., Beers, T. C., Lee, Y. S., et al. 2007, Nature, 450, 1020

Carney, B. W., Laird, J. B., Latham, D. W., \& Aguilar, L. A. 1996, AJ, 112, 668

Carretta, E. 2006, AJ, 131, 1766

Carretta, E., \& Gratton, R. 1997, A\&AS, 121, 95

Carretta, E., Cohen, J. G., Gratton, R. G., \& Behr, B. B. 2001, AJ, 122, 1469

Castelli, F., \& Kurucz, R. L. 2003, Modelling of Stellar Atmospheres, 210, 20P

Catelan, M., Ferraro, F. R., \& Rood, R. T. 2001, ApJ, 560, 970

Cayrel, R., Depagne, E., Spite, M., et al. 2004, A\&A, 416, 1117

Chapman, S. C., et al. 2007, ApJ, 662, L79

Chiba, M., \& Beers, T. C. 2000, AJ, 119, 2843

Cohen, J. G., \& Meléndez, J. 2005a, AJ, 129, 303

Cohen, J. G., \& Melendez, J. 2005b, AJ, 129, 1607

Côté, P., Djorgovski, S. G., Meylan, G., Castro, S., \& McCarthy, J. K. 2002, ApJ, 574,783

Cutri, R. M. 2003, Explanatory Supplement to the 2MASS All-Sky Data Release, http://www.ipac/caltech.edu/2mass/releases/allsky/ doc/explsup.html

Edvardsson, B., Andersen, J., Gustafsson, B., et al. 1993, A\&A, 275, 101

Frebel, A., Christlieb, N., Norris, J. E., et al. 2007, ApJ, 660, L117

Freeman, K., \& Bland-Hawthorn, J. 2002, ARA\&A, 40, 487

Fulbright, J. P. 2000, AJ, 120, 1841

Fulbright, J. P. 2002, AJ, 123, 404

Geisler, D., Wallerstein, G., Smith, V. V., \& Casetti-Dinescu, D. I. 2007, PASP, 119,939

Gilmore, G., Wilkinson, M. I., Wyse, R. F. G., et al. 2007, ApJ, 663, 948

Glatt, K., Gallagher, J. S., III, Grebel, E. K., et al. 2008, AJ, 135, 1106

Gonzalez, G., \& Wallerstein, G. 1998, AJ, 116, 765

Gratton, R. G., \& Sneden, C. 1988, A\&A, 204, 193

Gratton, R. G., \& Sneden, C. 1994, A\&A, 287, 927

Gratton, R., Sneden, C., \& Carretta, E. 2004, ARA\&A, 42, 385

Hanson, R. B., Sneden, C., Kraft, R. P., \& Fulbright, J. 1998, AJ, 116, 1286

Hartwick, F. D. A. 1987, NATO ASIC Proc. 207: The Galaxy, 281

Haynes, S., Burks, G., Johnson, C. I., \& Pilachowski, C. A. 2008, PASP, 120, 1097

Hilker, M. 2006, A\&A, 448, 171

Ivans, I. I., Sneden, C., \& Kraft, R. P. 1999, Ap\&SS, 265, 195

Ivans, I. I., Kraft, R. P., Sneden, C., et al. 2001, AJ, 122, 1438

Ivans, I. I., Sneden, C., James, C. R., et al. 2003, ApJ, 592, 906

Johnson, J. A. 2002, ApJS, 139, 219

Kelson, D. D. 2003, PASP, 115, 688

Kelson, D. D., Illingworth, G. D., van Dokkum, P. G., \& Franx, M. 2000, ApJ, 531,159
Kinman, T. D., Suntzeff, N. B., \& Kraft, R. P. 1994, AJ, 108, 1722

Kirby, E. N., Simon, J. D., Geha, M., Guhathakurta, P., \& Frebel, A. 2008, ApJ, $685, \mathrm{~L} 43$

Koch, A. 2009, AN, 330, 675

Koch, A., \& McWilliam, A. 2008, AJ, 135, 1551

Koch, A., McWilliam, A., Grebel, E. K., Zucker, D. B., \& Belokurov, V. 2008a, ApJ, 688, L13

Koch, A., Grebel, E. K., Gilmore, G. F., et al. 2008b, AJ, 135, 1580

Kraft, R. P., \& Ivans, I. I. 2003, PASP, 115, 143

Kraft, R. P., Sneden, C., Smith, G. H., Shetrone, M. D., \& Fulbright, J. 1998, AJ, 115,1500

Lee, J.-W., López-Morales, M., \& Carney, B. W. 2006, ApJ, 646, L119

Lee, Y.-W., Gim, H. B., \& Casetti-Dinescu, D. I. 2007, ApJ, 661, L49

Letarte, B., Hill, V., Jablonka, P., et al. 2006, A\&A, 453, 547

Magain, P. 1984, A\&A, 134, 189

Marcolini, A., Gibson, B. K., Karakas, A. I., \& Sánchez-Blázquez, P. 2009, MNRAS, 395, 719

McWilliam, A. 1998, AJ, 115, 1640

McWilliam, A., \& Bernstein, R. A. 2008, ApJ, 684, 326

McWilliam, A., \& Smecker-Hane, T. A. 2005, ApJ, 622, L29

McWilliam, A., Preston, G. W., Sneden, C., \& Searle, L. 1995, AJ, 109, 2757

McWilliam, A., Rich, R. M., \& Smecker-Hane, T. A. 2003, ApJ, 592, L21

Miceli, A., Rest, A., Stubbs, C. W., et al. 2008, ApJ, 678, 865

Nissen, P. E., \& Schuster, W. J. 1997, A\&A, 326, 751

Norris, J. E., \& Ryan, S. G. 1989, ApJ, 336, L17

Oke, J. B., Cohen, J. G., Carr, M., et al. 1995, PASP, 107, 375

Ortolani, S., \& Gratton, R. G. 1989, A\&AS, 79, 155

Palma, C., Majewski, S. R., \& Johnston, K. V. 2002, ApJ, 564, 736

Parmentier, G., \& Fritze, U. 2009, ApJ, 690, 1112

Pietrinferni, A., Cassisi, S., Salaris, M., \& Castelli, F. 2004, ApJ, 612, 168

Preston, G. W., Shectman, S. A., \& Beers, T. C. 1991, ApJ, 375, 121

Preston, G. W., Sneden, C., Thompson, I. B., Shectman, S. A., \& Burley, G. S. 2006, AJ, 132, 85

Pritzl, B. J., Venn, K. A., \& Irwin, M. 2005, AJ, 130, 2140

Prochaska, J. X., Naumov, S. O., Carney, B. W., McWilliam, A., \& Wolfe, A. M. 2000, AJ, 120, 2513

Qian, Y.-Z., \& Wasserburg, G. J. 2003, ApJ, 588, 1099

Ramírez, S. V., \& Cohen, J. G. 2003, AJ, 125, 224

Ramírez, I., \& Meléndez, J. 2005, ApJ, 626, 465

Reddy, B. E., Tomkin, J., Lambert, D. L., \& Allende Prieto, C. 2003, MNRAS, 340,304

Rutledge, G. A., Hesser, J. E., Stetson, P. B., et al. 1997a, PASP, 109, 883

Rutledge, G. A., Hesser, J. E., \& Stetson, P. B. 1997b, PASP, 109, 907

Ryan, S. G., Norris, J. E., \& Beers, T. C. 1996, ApJ, 471, 254

Sadakane, K., Arimoto, N., Ikuta, C., et al. 2004, PASJ, 56, 1041

Searle, L., \& Zinn, R. 1978, ApJ, 225, 357

Shetrone, M. D. 1996, AJ, 112, 1517

Shetrone, M. D., \& Keane, M. J. 2000, AJ, 119, 840

Shetrone, M. D., Côté, P., \& Sargent, W. L. W. 2001, ApJ, 548, 592

Shetrone, M., Venn, K. A., Tolstoy, E., et al. 2003, AJ, 125, 684

Sneden, C. A. 1973, Ph.D. Thesis, The University of Texas at Austin

Sneden, C., Pilachowski, C. A., \& Kraft, R. P. 2000, AJ, 120, 1351

Sohn, Y.-J., Park, J.-H., Rey, S.-C., et al. 2003, AJ, 126, 803

Stephens, A., \& Boesgaard, A. M. 2002, AJ, 123, 1647

Stetson, P. B., Bolte, M., Harris, W. E., et al. 1999, AJ, 117, 247

Takeda, Y., Zhao, G., Takada-Hidai, M., et al. 2003, Chinese Journal of Astronomy and Astrophysics, 3, 316

Takeda, Y., Kaneko, H., Matsumoto, N., Oshino, S., Ito, H., \& Shibuya, T. 2009, PASJ, in press [arXiv:0902.4504]

Thévenin, F., \& Idiart, T. P. 1999, ApJ, 521, 753

VandenBerg, D. A. 2000, ApJS, 129, 315

Venn, K. A., Irwin, M., Shetrone, M. D., Tout, C. A., Hill, V., \& Tolstoy, E. 2004, AJ, 128, 1177

Vogt, S. S., Allen, S. L., Bigelow, B. C., et al. 1994, Proc. SPIE, 2198, 362

Walker, M. G., Mateo, M., Olszewski, E. W., Bernstein, R., Sen, B., \& Woodroofe, M. 2007, ApJS, 171, 389

Wanajo, S., \& Ishimaru, Y. 2006, Nucl. Phys. A, 777, 676

Willman, B., Blanton, M. R., West, A. A., et al. 2005, AJ, 129, 2692

Winkler, H. 1997, MNRAS, 287, 481

Yong, D., \& Grundahl, F. 2008, ApJ, 672, L29

Yong, D., Grundahl, F., Nissen, P. E., Jensen, H. R., \& Lambert, D. L. 2005, A\&A, 438, 875

Zhang, H. W., Gehren, T., Butler, K., Shi, J. R., \& Zhao, G. 2006, A\&A, 457, 645

Zinn, R., \& West, M. J. 1984, ApJS, 55, 45

Zucker, D. B., Belokurov, V., Evans, N. W., et al. 2006, ApJ, 650, L41 